\title{
A New Form of the Circle Method, and its Application to Quadratic Forms
}

\author{
D.R. Heath-Brown \\ Magdalen College, Oxford
}

\section{Introduction}

The primary purpose of this paper is to describe a new version of the HardyLittlewood circle method. Before we begin with this it may be appropriate to remind the reader of the essential features of previous forms of the method, for which the reader is referred to the book of Vaughan [20]. The circle method starts with a generating function

$$
F(\alpha)=\sum_{n} r(n) e(\alpha n)
$$

where $e(x)$ is defined, as usual, to be $\exp (2 \pi i x)$. We then pick out the term $r(0)$ in which we are interested, by means of the formula

$$
r(0)=\int_{0}^{1} F(\alpha) d \alpha .
$$

If the coefficients $r(n)$ satisfy suitable arithmetic conditions the behaviour of $F(\alpha)$ will be determined by an appropriate rational approximation $a / q$ to $\alpha$, with small values of $q$ usually producing large values of $F(\alpha)$. When $\alpha$ lies in an interval $[a / q-\delta, a / q+\delta]$ with $q$ small, a 'major arc', one hopes to estimate $F(\alpha)$ asymptotically, while if the corresponding $q$ is large, for the 'minor arcs', one hopes that $F(\alpha)$ will be small, at least on average. One can distinguish two different types of application of the circle method. The first type, following the above pattern, uses one of a variety of mean-value techniques, Hua's inequality for example, to give an upper bound for the minor arc contribution. Results on Waring's problem are generally proved this way, for example. The second type of application, on which we shall be focusing our attention, avoids the minor arcs completely, and treats $F(\alpha)$ asymptotically for all $\alpha$. Here one cannot take advantage of the mean-value ideas used in the first type of problem. The savings come instead from the availability of good estimates for 'complete' exponential sums. Kloosterman's work [12] on quaternary quadratic forms, and the papers of the author [5] and Hooley [8] and [9] on cubic forms, are of this latter type. We should point out that such applications do not include any problems in which $F$ is the generating function for a polynomial of degree 4 or more. There are 
also very few applications involving prime numbers, a notable exception being the work on

$$
\liminf _{n \rightarrow \infty} \frac{p_{n+1}-p_{n}}{\log n}
$$

initiated by Hardy and Littlewood in their unpublished 1926 manuscript 'Some problems of Partitio Numerorum VII'. Moreover it does not yet seem possible to work in this way with questions concerning two-dimensional generating functions, in which one would consider

$$
\int_{0}^{1} \int_{0}^{1} \sum_{m, n} r(m, n) e(\alpha m+\beta n) d \alpha d \beta .
$$

However, where this second method is available, it allows more delicate arguments to be brought into play. In particular it is possible, following the work of Kloosterman cited above, to introduce non-trivial averaging over the numerators $a$ of the approximating fractions $a / q$. We shall refer to such averaging processes as the 'Kloosterman refinement'. One can, indeed, average non-trivially with respect to $q$, although this has been successfully accomplished only in the work of Hooley [8]. Such an argument may be termed a 'double Kloosterman refinement'. There are considerable technical difficulties in performing all the averaging processes that can potentially produce savings in the method. As an example, we quote, in modified form, the version of the circle method used in the author's work [5; Lemma 7].

For any $Q \geq 1$ we have

$$
\begin{aligned}
\int_{0}^{1} F(\alpha) d \alpha= & \sum_{q \leq Q} \int_{-1 / q Q}^{1 / q Q} F_{0}(q ; \alpha) d \alpha \\
& +O\left\{Q^{-2} \sum_{q \leq Q} \sum_{|u| \leq q / 2}(1+|u|)^{-1} \max _{1 / 2 \leq q Q|\alpha| \leq 1}\left|F_{u}(q ; \alpha)\right|\right\},
\end{aligned}
$$

where

$$
F_{u}(q ; \alpha)=\sum_{\substack{a=1 \\(a, q)=1}}^{q} e\left(\frac{\bar{a} u}{q}\right) F\left(\frac{a}{q}+\alpha\right),
$$

with $\bar{a}$ denoting the inverse modulo $q$.

The occurence here of an exponential sum involving $\bar{a}$ seems to be typical of attempts to average over $a$, and indeed Kloosterman sums originally arose in just this context. While the above result has the potential for averaging over $q$ in the main term, the modulus signs on $F$ in the error term preclude any great savings in the latter. Our first goal, in Theorem 2, is a result which plays the same rôle as that above, but in which only the main term is present. Indeed the saving that comes from the integration over $\alpha$ will be incorporated into the estimates automatically. 
We begin with a result which is essentially due to Duke, Friedlander and Iwaniec [4].

Theorem 1 For any integer $n$ let

$$
\delta_{n}= \begin{cases}1, & n=0 \\ 0, & n \neq 0\end{cases}
$$

Then for any $Q>1$ there is positive constant $c_{Q}$, and an infinitely differentiable function $h(x, y)$ defined on the set $(0, \infty) \times \mathbb{R}$, such that

$$
\delta_{n}=c_{Q} Q^{-2} \sum_{q=1}^{\infty} \sum_{a(\bmod q)}{ }^{*} e_{q}(\operatorname{an}) h\left(\frac{q}{Q}, \frac{n}{Q^{2}}\right) .
$$

The constant $c_{Q}$ satisfies

$$
c_{Q}=1+O_{N}\left(Q^{-N}\right)
$$

for any $N>0$. Moreover $h(x, y) \ll x^{-1}$ for all $y$, and $h(x, y)$ is non-zero only for $x \leq \max (1,2|y|)$.

Here we have introduced the notation $e_{q}(x)$ for $e(x / q)$. Moreover, here, as later, $\Sigma^{*}$ indicates a summation for residues $a$ with $(a, q)=1$.

One may find alternative formulae for $\delta_{n}$ in the literature, as in Iwaniec [11] for example, but in many of these, exponential sums involving $\bar{a}$ appear, making them unsuitable for our purposes. These expressions for $\delta_{n}$ have been described as giving forms of the circle method. However, where applications have been given, they have not been to problems of the classical type, containing a main term given by a singular series. Our aim here is to show how Theorem 1 may be applied to the normal range of circle method problems.

To see how Theorem 1 relates to the usual circle method we observe that the summations over $q$ and $a$ in the theorem play exactly the same rôle as when one writes

$$
\delta_{n}=\int_{0}^{1} e(\alpha n) d \alpha
$$

and decomposes the range of integration into major arcs around the points $a / q$. In particular one may observe that only values $q \ll Q$ occur, providing that $n \ll Q^{2}$. This corresponds to using Dirichlet's approximation theorem to produce values $q \leq Q$. It would be nice to have a two-dimensional version of Theorem 1, corresponding to Dirichlet's theorem in two dimensions. This might perhaps involve a sum of the form

$$
\sum_{a, b(\bmod q)} e_{q}(a m+b n) h\left(\frac{q}{Q^{3 / 2}}, \frac{m}{Q^{2}}, \frac{n}{Q^{2}}\right) .
$$

One normally applies the circle method to search for solutions $\mathbf{x} \in Z^{n}$ of a polynomial equation $F(\mathbf{x})=0$ with $F\left(X_{1}, \ldots, X_{n}\right) \in \mathbb{Z}\left[X_{1}, \ldots, X_{n}\right]$. In 
general one confines the search to some bounded subset $\mathcal{R}$ of $\mathbb{R}^{n}$. We shall consider a slightly more general situation in which one has a smooth bounded function $w(\mathbf{x})$ compactly supported in $\mathbb{R}^{n}$. Thus one could take $w(\mathbf{x})$ to be an approximation to the characteristic function of $\mathcal{R}$ if one so wished. Our aim is to estimate

$$
N^{(0)}(F, w)=\sum w(\mathbf{x}),
$$

the sum being taken over all $\mathbf{x} \in \mathbb{Z}^{n}$ for which $F(\mathbf{x})=0$. Theorem 1 then leads to the following.

\section{Theorem 2 We have}

$$
N^{(0)}(F, w)=c_{Q} Q^{-2} \sum_{q=1}^{\infty} \sum_{a(\bmod q)} * \sum_{\mathbf{x}} w(\mathbf{x}) e_{q}(a F(\mathbf{x})) h\left(\frac{q}{Q}, \frac{F(\mathbf{x})}{Q^{2}}\right)
$$

for any $Q>1$. Moreover, if $w$ is infinitely differentiable then

$$
N^{(0)}(F, w)=c_{Q} Q^{-2} \sum_{\mathbf{c} \in \mathbb{Z}^{n}} \sum_{q=1}^{\infty} q^{-n} S_{q}(\mathbf{c}) I_{q}^{(0)}(\mathbf{c}),
$$

where

$$
S_{q}(\mathbf{c})=\sum_{a(\bmod q)} * \sum_{\mathbf{b}(\bmod q)} e_{q}(a F(\mathbf{b})+\mathbf{c} \cdot \mathbf{b})
$$

and

$$
I_{q}^{(0)}(\mathbf{c})=\int_{\mathbb{R}^{n}} w(\mathbf{x}) h\left(\frac{q}{Q}, \frac{F(\mathbf{x})}{Q^{2}}\right) e_{q}(-\mathbf{c} . \mathbf{x}) d \mathbf{x} .
$$

The statement of the theorem introduces some notation which will be standard throughout this paper: a sum with a condition $\mathbf{b} \bmod q$ ) will mean that all components of $\mathbf{b}$ will run from 0 to $q-1$, and an integral $\int f(\mathbf{x}) d \mathbf{x}$ will be the $n$-fold repeated integral over $\mathbb{R}^{n}$.

The expression (1.2) may be compared with the relation (4.1) of the author's work [5] on cubic forms. In the case of interest the latter states that

$$
\sum_{a(\bmod q)}^{*} \sum_{\mathbf{x} \in \mathbb{Z}^{n}} e\left\{\left(\frac{a}{q}+\alpha\right) F(\mathbf{x})\right\}=q^{-n} \sum_{\mathbf{c} \in \mathbb{Z}^{n}} S_{q}(\mathbf{c}) I_{q}^{(1)}(\mathbf{c}),
$$

where

$$
I_{q}^{(1)}(\mathbf{c})=\int_{\mathbb{R}^{n}} w(\mathbf{x}) e\left(\alpha F(\mathbf{x})-\frac{\mathbf{c} \cdot \mathbf{x}}{q}\right) d \mathbf{x} .
$$

One may think of Theorem 2 as having had an integration over $\alpha$ performed, to produce a factor $h\left(q / Q, F(\mathbf{x}) / Q^{2}\right)$. Both expressions result from applications of the Poisson summation formula, and in each case the usefulness of the result depends on the size of the vectors $\mathbf{c}$ which must be considered. It turns out that the range for $\mathbf{c}$ grows as the degree of the polynomial $F$ increases, in such a way that one cannot hope for an asymptotic formula for $N^{(0)}(F, w)$ when $F$ has degree 4 or more. This assumes, as is the case at present, that one 
cannot average over c non-trivially. It follows that our method is likely to be of interest for polynomials of degree 2 and 3 only, although it is conceivable that worthwhile upper bounds for $N^{(0)}(F, w)$ could be obtained in other cases. In the present paper we shall develop the theory for general degree where this can be done without too much effort. We shall then apply the result to give a thorough treatment of the quadratic case. It is our intention to examine cubic forms in a later paper.

It should perhaps be mentioned that Hooley [6] and [7] has given another argument that produces what may be regarded as a form of the circle method. This, in the same way as Theorem 2 , avoids sums involving $\bar{a}$. The method applies only in certain very special cases, principally to polynomials of the form

$$
x_{1}^{2}+x_{2}^{2}-H\left(x_{3}, \ldots, x_{n}\right) .
$$

One begins by using the formula

$$
\#\left\{x_{1}^{2}+x_{2}^{2}=H\right\}=4 \sum_{d \mid H} \chi(d)=4 \sum_{q \mid H, q \leq Q} \chi(q)+4 \sum_{q|H, q<| H \mid / Q} \chi(|H| / q),
$$

where $\chi$ is the non-principal character modulo 4 , so that the problem is reduced to questions concerning

$$
\left\{\left(x_{3}, \ldots, x_{n}\right): q \mid H\left(x_{3}, \ldots, x_{n}\right)\right\} .
$$

In certain circumstances the method can also be adapted for use with polynomials of the form

$$
x_{1}^{2}-H\left(x_{2}, \ldots, x_{n}\right) .
$$

Indeed, as is hinted at by Hooley [7; page 95] his method may be iterated so as to mimic Weyl's estimation of the exponential sum $\sum_{n} e\left(\alpha n^{k}\right)$. This enables appropriate problems involving higher degree polynomials to be handled. It should be clear however that Theorem 2 gives a much more general approach. While Hooley applies his method to polynomials of arbitrary degree, we have said that our approach is most useful for degrees at most 3 . This is because we have used the Poisson summation formula to pass from (1.1) to (1.2). If one works with (1.1) higher degrees can be handled, but the advantage over previous forms of the circle method is much less.

In practice the problems in which one is usually interested are of two types. In the first of these the polynomial $F$ is a fixed form of degree $d$ in $n$ variables, and one looks for integer zeros in a region $P \mathcal{R}$, where $\mathcal{R}$ is a fixed bounded subset of $\mathbb{R}^{n}$ and $P \rightarrow \infty$. This corresponds to finding rational points on a projective hypersurface. In the second type of problem $F$ is of the shape $F^{(0)}(\mathbf{x})-m$, where $F^{(0)}$ is a fixed form of degree $d$ in $n$ variables, and $m$ is a variable integer. Here one seeks integer solutions $\mathbf{x}$ of $F^{(0)}(\mathbf{x})=m$ in an expanding region $P \mathcal{R}$ as before, but now it is natural to take $P=|m|^{1 / d}$. This is the category which Waring's problem, with $F^{(0)}(\mathbf{x})=\sum x_{j}^{d}$, comes into. With no loss of generality we shall take $m>0$ in this case. There is a third type of problem, analogous to the second case above, but with $m$ fixed. There is no difficulty in handling this 
situation, and indeed it is simpler than the second problem. However we shall not consider it further in this paper.

We therefore make the following conventions. The expanding region will correspond to using a weight $w\left(P^{-1} \mathbf{x}\right)$, where $w$ is fixed and $P \rightarrow \infty$. Instead of investigating $N^{(0)}(F, w)$ we shall consider

$$
N(F, w)=N(F, w, P)=\sum w\left(P^{-1} \mathbf{x}\right),
$$

the sum being taken over all $\mathbf{x} \in \mathbb{Z}^{n}$ for which $F(\mathbf{x})=0$, as before. Theorem 2 will then apply to $N(F, w)$, with $I_{q}^{(0)}(\mathbf{c})$ replaced by

$$
I_{q}(\mathbf{c})=\int_{\mathbb{R}^{n}} w\left(P^{-1} \mathbf{x}\right) h\left(\frac{q}{Q}, \frac{F(\mathbf{x})}{Q^{2}}\right) e_{q}(-\mathbf{c} \cdot \mathbf{x}) d \mathbf{x} .
$$

The polynomial $F$ will have degree $d$ and $n$ variables. It will either be a form, in which case it will be fixed throughout the paper, or it will take the shape $F(\mathbf{x})=F^{(0)}(\mathbf{x})-m$, where $F^{(0)}$ is a fixed form and $m$ tends to infinity. In the latter case we shall take $P=m^{1 / d}$. When $F$ is a form we shall define $F^{(0)}=F$. We shall set $G=F$ in the case in which $F$ is a form, and $G=F^{(0)}-1$ otherwise, so that $G(\mathbf{x})=P^{-d} F(P \mathbf{x})$ in both cases.

In order to analyse the 'singular integral' we shall impose a non-singularity condition, namely that $\nabla G(\mathbf{x}) \neq \mathbf{0}$ on the closure of $\operatorname{supp}(w)$. When $F(\mathbf{x})=$ $F^{(0)}(\mathbf{x})-m$ with $m \neq 0$ one sees that if $\nabla G(\mathbf{x})=\mathbf{0}$ then

$$
0=\mathbf{x} \cdot \nabla F^{(0)}(\mathbf{x})=d F^{(0)}(\mathbf{x})=d(G(\mathbf{x})+1) .
$$

Thus if $w$ is chosen so that $|G(\mathbf{x})| \leq 1 / 2$ on $\operatorname{supp}(w)$, then the non-singularity condition holds automatically. It is therefore apparent that the condition is only of significance when $F$ is itself a form of degree $d$. In the latter case our condition ensures that the closure of $\operatorname{supp}(w)$ cannot include the origin. We shall assume henceforth that the non-singularity condition holds for our weights $w$, without further comment.

In order to introduce a degree of uniformity into our results we shall require the weight $w$ to belong to the class $\mathcal{C}(S)$, which we shall describe in $\S 2$. Here $S$ is a list of parameters which may be numbers, sets or functions, and which include amongst them the function $G$, and a number $\lambda>0$ such that $|\nabla G| \geq \lambda$ on $\operatorname{supp}(w)$. It will become apparent in $\S 2$ that $w \in \mathcal{C}(w, G, \lambda)$ for any infinitely differentiable function of compact support. Indeed if $|\nabla G|>0$ on $\operatorname{supp}(w)$ then there will be a suitable $\lambda$ depending only on $G$ and $w$. It follows that whenever there is a dependence on $S$ in our results this can be replaced by a dependence on $w$ and $G$.

It turns out that the singular integral is most readily described when $w$ belongs to a more restricted class of functions, denoted by $\mathcal{C}_{0}(S)$. The definition of the sets $\mathcal{C}_{0}$ is somewhat complicated and will not be given until $\S 6$. However, two important properties of functions $w$ in these sets should be mentioned at this point. Firstly $\partial G / \partial x_{1}$ is positive and bounded away from zero on $\operatorname{supp}(w)$, and secondly for each $\left(x_{2}, \ldots, x_{n}\right)$ in an appropriate set there is a corresponding 
unique solution of $G\left(x_{1}, x_{2}, \ldots, x_{n}\right)=0$. We shall show, in $\S 6$, how the problem of the 'singular integral' for general weights can be reduced to that in which $w \in \mathcal{C}_{0}(S)$. This will allow us to give the following description of the singular integral.

Theorem 3 Suppose that $w$ is infinitely differentiable, and has compact support. Suppose further that $\nabla G(\mathbf{x}) \neq \mathbf{0}$ on the closure of $\operatorname{supp}(w)$. Then the limit

$$
\sigma_{\infty}=\sigma_{\infty}(G, w)=\lim _{\varepsilon \rightarrow 0}(2 \varepsilon)^{-1} \int_{|G(\mathbf{x})| \leq \varepsilon} w(\mathbf{x}) d \mathbf{x}
$$

exists. If, further, $w$ is real-valued and non-negative everywhere, and takes a strictly positive value for some real solution $\mathbf{x}$ of $G(\mathbf{x})=0$, then $\sigma_{\infty}>0$.

If moreover $w \in \mathcal{C}_{0}(S)$ for some set $S$, then

$$
\sigma_{\infty}=\int \ldots \int w(\mathbf{x}) \frac{d x_{2} d x_{3} \ldots d x_{n}}{\partial G / \partial x_{1}},
$$

where $x_{1}$ is determined by the condition $G(\mathbf{x})=0$. The integral here is over vectors $\mathbf{y}=\left(x_{2}, \ldots, x_{n}\right)$ for which there is at least one $x$ with $(x, \mathbf{y}) \in \operatorname{supp}(w)$.

It is appropriate to mention here that the corresponding $p$-adic density of solutions is given by

$$
\sigma_{p}=\lim _{k \rightarrow \infty} p^{-(n-1) k} \#\left\{\mathbf{x}\left(\bmod p^{k}\right): F(\mathbf{x}) \equiv 0\left(\bmod p^{k}\right)\right\} .
$$

Conditions under which this limit exists will become apparent in $\S 11$. The corresponding 'singular series' will be

$$
\prod_{p} \sigma_{p}
$$

which may converge only conditionally, if at all.

We are now ready to state our results on problems involving quadratic forms. A great many methods have been used in the past to investigate such questions, and we do not claim any great degree of novelty for our results. Indeed Malyshev [13] has investigated sums very much like $N(F, w)$, for general quadratic polynomials $F$, and a weight $w(\mathbf{x})$ which is a negative exponential in a quadratic function of $\mathbf{x}$. However for homogeneous $F$ his results only cover the case $n \geq 5$, whereas we shall be able to handle all $n \geq 3$. We hope that our approach will be of methodological interest, and that it will serve as a pointer to the situation for polynomials of higher degree.

Our first theorem describes the equation $F^{(0)}(\mathbf{x})=m$, where $F^{(0)}$ is a nonsingular quadratic form.

Theorem 4 Let $n \geq 4$ and let $F=F^{(0)}-m$, where $F^{(0)}$ is a non-singular quadratic form in $n$ variables. Suppose that $w \in \mathcal{C}(S)$ for some set of parameters S. Then

$$
N(F, w)=\sigma_{\infty}(G, w) \sigma\left(F^{(0)}, m\right) m^{n / 2-1}+O_{S, \varepsilon}\left(m^{(n-1) / 4+\varepsilon}\right),
$$


where $\sigma\left(F^{(0)}, m\right)$ is the usual Hardy-Littlewood singular series. In particular if $n \geq 5$ and $F^{(0)}=m$ is solvable in every $p$-adic field then $1 \ll \sigma\left(F^{(0)}, m\right) \ll 1$, the implied constants depending only on $F^{(0)}$. In the alternative case $\sigma\left(F^{(0)}, m\right)$ vanishes. If $n=4$ we will have $m^{-\varepsilon} \ll_{\varepsilon} \sigma\left(F^{(0)}, m\right) \ll_{\varepsilon} m^{\varepsilon}$ providing that $F^{(0)}(\mathbf{x})=m$ is solvable in every $p$-adic field, with $p \nmid \nabla F^{(0)}(\mathbf{x})$.

The proof uses a Kloosterman refinement. An asymptotic formula could have been obtained without this (but with a worse error term) for $n \geq 5$, but not for $n=4$. That non-singular $p$-adic solutions are required when $n=4$ is shown by the example $x_{1}^{2}+x_{2}^{2}+7\left(x_{3}^{2}+x_{4}^{2}\right)=3.7^{2 u}$ (see Watson [21; page 121]) for which there are no integer solutions, even though the equation is everywhere locally solvable.

By using a particular weight function $w(\mathbf{x})$ we may deduce the following.

Corollary 1 Let $n \geq 4$ and let $F^{(0)}$ be a positive-definite quadratic form in $n$ variables. Then

$$
\#\left\{\mathbf{x} \in \mathbb{Z}^{n}: F^{(0)}(\mathbf{x})=m\right\}=\sigma_{\infty}(G) \prod_{p} \sigma\left(F^{(0)}, m\right) m^{n / 2-1}+O\left(m^{(n-1) / 4+\varepsilon}\right) .
$$

where the implied constant depends only on $F^{(0)}$ and $\varepsilon$. Here $\sigma_{\infty}(G)$ is the singular integral in its usual form, which can be expressed as

$$
\sigma_{\infty}(G)=\lim _{\varepsilon \rightarrow 0}(2 \varepsilon)^{-1} \int_{|G(\mathbf{x})| \leq \varepsilon} d \mathbf{x} .
$$

As with Theorem 4 , the case $n \geq 5$ of this result (with a worse error term) is easily obtained by previous forms of the circle method. When $n=4$ the result was first obtained (again with a worse error term) by Kloosterman [12], via the Kloosterman refinement. Modern forms of the 'traditional' circle method, again using the Kloosterman refinement, would, however, yield the exponent $(n-1) / 4+\varepsilon$ for all $n \geq 4$, just as in Corollary 1 . While the use of Theorem 2 does not itself bring in exponential sums involving $\bar{a}$, in contrast to Kloosterman's approach, we should stress that Kloosterman sums none the less occur in the analysis. Their presence is linked to quadratic forms in an even number of variables, rather than to the end points of the Farey dissection.

The error term in Theorem 4 is better than one would expect for a corresponding unweighted counting function. However for definite forms the unweighted version arises quite naturally. The corollary may be proved by a completely different method, using modular functions. The exponent in the error term can then be improved to $(n-2) / 4+\varepsilon$, for even values of $n$, by using the Ramanujan-Petersson conjecture, as proved by Deligne [1]. For odd values of $n$ the corresponding conjecture remains undecided, but some improvement in the exponent is available by virtue of the results of Iwaniec [10] and Duke [2]. Indeed their methods allow one to handle the case $n=3$, which we have not touched. The interested reader should see the papers by Duke [3] and Moroz [15] for further details. If one attempts to apply Theorem 2 to the case $n=3$ one 
eventually sees that the problem reduces to the estimation of certain averages of Salié sums, and it is exactly these averages which form the starting point for Iwaniec's work [10].

The results in the homogeneous case are naturally more delicate than those we have looked at so far. However it turns out that we are able to give a complete proof of the Hasse principle for rational quadratic forms in $n \geq 3$ variables.

Previous approaches to the Hasse principle hace been of broadly two types. In the first line of attack one begins by establishing the case $n=3$ by one of a variety of elementary methods, and then proceeds to the case $n=4$ using Dirichlet's theorem on primes in arithmetic progression in conjunction with the product formula for Hilbert symbols. The cases $n \geq 5$ then follow fairly easily from the result for $n=4$. This is the procedure used by Mordell 114; Chapter 7 ], for example. The second line of attack begins by establishing the case $n=3$ over an arbitrary quadratic number field, using class field theory. One can then deduce the results for $n=4$ and $n \geq 5$ in a relatively straightforward manner. This is the approach used by O'Meara [16].

Our line of attack is quite clearly distinct from either of these methods. We begin with the easiest case. In the statements which follow we shall not attempt to produce the best possible error terms that our method allows.

Theorem 5 Let $n \geq 5$ and let $F$ be a non-singular quadratic form in $n$ variables. Suppose that $w \in \mathcal{C}(S)$ for some set of parameters $S$. Then

$$
N(F, w)=\sigma_{\infty}(F, w) \sigma(F) P^{n-2}+O_{S, \varepsilon}\left(P^{(n-1+\delta) / 2+\varepsilon}\right),
$$

where $\delta=0$ or 1 , according as $n$ is odd or even. Here $\sigma_{\infty}(F, w)$ and $\sigma(F)$ are the singular integral and the singular series respectively. If, further, $w$ is real-valued and non-negative everywhere, and takes a strictly positive value for some real solution $\mathbf{x}$ of $F(\mathbf{x})=0$, then $\sigma_{\infty}(F, w)>0$. In contrast $\sigma(F)$ is always positive.

Here no Kloosterman refinement is necessary for an asymptotic formula to be obtained. However our proof does use such an argument, thereby getting a better error term than would otherwise be available. In contrast, when $n=4$ or 3 a double Kloosterman refinement is necessary in order to produce a satisfactory asymptotic formula.

The next case to consider, namely $n=4$, is already one which has not previously been tackled by the circle method. Indeed in most treatments of the Hasse-Minkowski Theorem it is the most difficult step. One sees that the non-vanishing of a Dirichlet $L$-function $L(1, \chi)$ is naturally brought into the question. However the form of the result makes it easy to deduce that $L(1, \chi)$ is indeed positive.

Theorem 6 Let $F$ be a non-singular quadratic form in 4 variables, whose determinant is not a square. Suppose that $w \in \mathcal{C}(S)$ for some set of parameters S. Then

$$
N(F, w)=\sigma_{\infty}(F, w) L(1, \chi) \sigma^{*}(F) P^{2}+O_{S, \varepsilon}\left(P^{3 / 2+\varepsilon}\right) .
$$


Here the character $\chi$ is the Jacobi symbol $\left(\frac{\operatorname{det}(F)}{*}\right)$ and

$$
\sigma^{*}(F)=\prod_{p} \sigma_{p}^{\prime}
$$

where

$$
\sigma_{p}^{\prime}=\left(1-\chi(p) p^{-1}\right) \sigma_{p} .
$$

The singular integral $\sigma_{\infty}(F, w)$ is positive under the same conditions as in Theorem 5, while $\sigma^{*}(F)>0$ providing that $F=0$ has non-trivial solutions in every $p$-adic field.

If we put $D=\operatorname{det}(F)$, we may apply the above result to the form

$$
F^{*}=2 x_{1} x_{2}+x_{3}^{2}-D x_{4}^{2},
$$

which also has determinant $D$. It can be shown that this form has $\gg P^{2}$ solutions in the annulus $P \ll|\mathbf{x}| \ll P$, given by

$$
\begin{aligned}
& x_{1}=y_{1}^{2}-D y_{2}^{2}, \\
& x_{2}=2 D y_{4}^{2}-2 y_{3}^{2}, \\
& x_{3}=2 y_{1} y_{3}+2 D y_{2} y_{4}, \\
& x_{4}=2 y_{1} y_{4}-2 y_{2} y_{3},
\end{aligned}
$$

for $P^{1 / 2} \ll|\mathbf{y}| \ll P^{1 / 2}$. Using a suitable weight $w$ we can then deduce from Theorem 6 that $L(1, \chi)$ must be positive. This gives a completely new proof of the non-vanishing of $L(1, \chi)$. Unfortunately however when the argument is made precise one obtains only the weak lower bound $L(1, \chi) \gg|D|^{-1}$.

The remaining cases reveal some new features.

Theorem 7 Let $F$ be a non-singular quadratic form in 4 variables, whose determinant is a square. Suppose that $w \in \mathcal{C}(S)$ for some set of parameters $S$. Then there is a constant $\sigma_{1}(F, w)$ depending on $F$ and $w$ only, such that

$$
N(F, w)=\sigma_{\infty}(F, w) \sigma^{*}(F) P^{2} \log P+\sigma_{1}(F, w) P^{2}+O_{S, \varepsilon}\left(P^{3 / 2+\varepsilon}\right) .
$$

Here

$$
\sigma^{*}(F)=\prod_{p} \sigma_{p}^{\prime}
$$

with

$$
\sigma_{p}^{\prime}=\left(1-p^{-1}\right) \sigma_{p}
$$

The singular integral $\sigma_{\infty}(F, w)$ is positive under the same conditions as in Theorem 5, while $\sigma^{*}(F)>0$ providing that $F=0$ has non-trivial solutions in every $p$-adic field. 
Theorem 8 Let $F$ be a non-singular quadratic form in 3 variables. Suppose that $w \in \mathcal{C}(S)$ for some set of parameters $S$. Then there is a constant $\sigma_{1}(F, w)$ depending on $F$ and $w$ only, such that

$$
N(F, w)=\frac{1}{2} \sigma_{\infty}(F, w) \sigma^{*}(F) P \log P+\sigma_{1}(F, w) P+O_{S, \varepsilon}\left(P^{5 / 6+\varepsilon}\right) .
$$

Here

$$
\sigma^{*}(F)=\prod_{p} \sigma_{p}^{\prime}
$$

with

$$
\sigma_{p}^{\prime}=\left(1-p^{-1}\right) \sigma_{p}
$$

The singular integral $\sigma_{\infty}(F, w)$ is positive under the same conditions as in Theorem 5, while $\sigma^{*}(F)>0$ providing that $F=0$ has non-trivial solutions in every p-adic field.

We remark that the constant $\sigma_{1}(F, w)$, while it is given explicitly by the proof, does not occur in a form which has a natural interpretation. However, in the case $n=3$, the constant appears to measure the effect of small solutions of $F=0$. Thus if $\mathbf{x}_{0}$ is any fixed non-trivial solution of $F=0$, then the multiples $k \mathbf{x}_{0}$ contribute $c P$ to $N(F, w)$, for a positive constant $c$, depending on $\mathbf{x}_{0}$ and $w$. On the other hand, as we shall see in Corollary 2 below, if one counts only primitive solutions of $F=0$, the constant $\sigma_{1}(F, w)$ disappears. One further comment of interest is that, while $\sigma(F)$ and $\sigma^{*}(F)$ arise solely from the term $\mathbf{c}=\mathbf{0}$ in (1.2), certain other values of $\mathbf{c}$ contribute to $\sigma_{1}(F, w)$, as we shall see in $\S 13$.

It is very unusual with the circle method to find that the leading term is not merely a power of $P$. The simplest heuristic estimate for $N(F, w)$ (ignoring $p$-adic effects) would suggest a size of order $P^{n-2}$. However in Theorems 7 and 8 the $p$-adic densities have a systematic bias which makes $\prod \sigma_{p}$ diverge to infinity. The extra factor $\log P$ may be thought of as allowing for this effect. The results above are not quite the first of this type. In fact Siegel [17] obtained a result corresponding to Theorem 7 for a certain infinite weighted sum, also using the circle method.

A simple example corresponding to Theorem 7 is given by the equation

$$
x_{1}^{2}+x_{2}^{2}=x_{3}^{2}+x_{4}^{2}
$$

in the region

$$
P^{2}<x_{1}^{2}+x_{2}^{2}, x_{3}^{2}+x_{4}^{2} \leq 2 P^{2} .
$$

The (unweighted) number of solutions is given by

$$
\sum_{P^{2}<n \leq 2 P^{2}} r^{2}(n),
$$

where $r(n)$ counts representations an a sum of two squares. The corresponding Dirichlet series $\sum_{n=1}^{\infty} r^{2}(n) n^{-s}$ has a double pole at $s=1$, so that the number of solutions is of exact order $P^{2} \log P$, as predicted by Theorem 7 . 
In Theorems 5-8 it is easy to translate between $N(F, w)$ and the corresponding counting function for primitive solutions. Thus, if we write

$$
N_{\text {prim }}(F, w)=N_{\text {prim }}(F, w, P)=\sum \operatorname{prim} w\left(P^{-1} \mathbf{x}\right),
$$

the sum being over all primitive vectors $\mathbf{x}$ for which $F(\mathbf{x})=0$, then

$$
N(F, w, P)=\sum_{k=1}^{\infty} N_{\text {prim }}(F, w, P / k)
$$

and

$$
N_{\text {prim }}(F, w, P)=\sum_{k=1}^{\infty} \mu(k) N(F, w, P / k) .
$$

It follows that Theorems 5 and 6 lead to equivalent results for $N_{\text {prim }}(F, w)$, in which the only change is that the leading term is divided by $\zeta(n-2)$. Similarly in Theorem 7 the corresponding result has the leading term, involving $P^{2} \log P$, divided by $\zeta(2)$, while the constant $\sigma_{1}(F, w)$ is replaced by a different value $\sigma_{1}^{\prime}(F, w)$, say. However for Theorem 8 we have the following corollary.

Corollary 2 Let $F$ be a non-singular quadratic form in 3 variables. Suppose that $w \in \mathcal{C}(S)$ for some set of parameters $S$. Then there is a positive absolute constant $c$, such that

$$
N_{\text {prim }}(F, w)=\frac{1}{2} \sigma_{\infty}(F, w) \sigma^{*}(F) P+O_{S}(P \exp \{-c \sqrt{\log P}\}),
$$

with $\sigma^{*}(F)$ as in Theorem 8.

Since $\zeta^{-1}(n-2)=\prod\left(1-p^{2-n}\right)$ we now see that

$$
N_{\text {prim }}(F, w, P) \sim P^{n-2} \sigma_{\infty}(F, w) \prod_{p}\left(1-p^{2-n}\right) \sigma_{p},
$$

for the cases covered by Theorems 5 and 6 . However for $n=3$, Corollary 2 yields

$$
N_{\text {prim }}(F, w, P) \sim \frac{1}{2} P^{n-2} \sigma_{\infty}(F, w) \prod_{p}\left(1-p^{2-n}\right) \sigma_{p} .
$$

The product over primes has a natural interpretation, since

$$
\left(1-p^{2-n}\right) \sigma_{p}=\lim _{k \rightarrow \infty} p^{-(n-1) k} \#\left\{\mathbf{x}\left(\bmod p^{k}\right): p \nmid \mathbf{x}, F(\mathbf{x}) \equiv 0\left(\bmod p^{k}\right)\right\},
$$

which we can think of as being the $p$-adic density for primitive vectors. It therefore remains to understand the appearance of the factor $\frac{1}{2}$ in the case $n=3$, which can be thought of as corresponding to a Tamagawa number of 2 . In the proof of Theorem 8 this factor arises from the residue at $s=0$ of

$$
\zeta(2 s+1) \frac{P^{s}}{s} .
$$


Here we have $\zeta(2 s+1)$ rather than, say, $\zeta(s+1)$, because in forming the local densities from the sums $S_{p^{k}}(\mathbf{0})$ it is the terms with $k=2$ rather than $k=1$ which dominate.

Although we shall not provide any details it is not hard to see that we can handle the modified counting function

$$
\sum_{\substack{F(\mathbf{x})=0 \\ \mathbf{x} \equiv \mathbf{k}(\bmod m)}} w\left(P^{-1} \mathbf{x}\right),
$$

where $\mathbf{x}$ is restricted to lie in a fixed congruence class, in exactly the same way as we do for $N(F, w)$. In this way we can prove results on 'weak approximation'. For example Theorems 5-8 may be modified to show the following.

Corollary 3 Let $F$ be a non-singular rational quadratic form in $n \geq 3$ variables, and suppose $F=0$ has a non-trivial zero in every completion of $\mathbb{Q}$. Let $\mathcal{S}$ be a finite set of primes, and for each $p \in \mathcal{S}$ let $\mathbf{x}_{p}$ be a non-trivial zero of $F$ in $Z_{p}$. Similarly let $\mathbf{x}_{\infty}$ be a non-trivial real zero of $F$. Then for any $\varepsilon>0$, and for any sufficiently large $P$, there exists an integral zero $\mathbf{x}$ of $F$ with $\left|P^{-1} \mathbf{x}-\mathbf{x}_{\infty}\right|<\varepsilon$ and $\left|\mathbf{x}-\mathbf{x}_{p}\right|_{p}<\varepsilon$ for all $p \in \mathcal{S}$.

If we try to do the same thing for primitive integer vectors it turns out that an analogue of the above can be readily proved when $n \geq 4$, but that the process that leads to Corollary 2 cannot be generalized when a congruence restriction is placed on $\mathbf{x}$. It is instructive to see why this is so. In the deduction of Corollary 2 from Theorem 8 the constant $\sigma_{1}(F, w)$ disappears, because it becomes multiplied by

$$
\sum_{d=1}^{\infty} \frac{\mu(d)}{d}=0 .
$$

However if we wish to impose a condition $\mathbf{x} \equiv \mathbf{k} \bmod m)$ the analogous sum will be of the form

$$
\sum_{\substack{d=1 \\ d \equiv d_{0}(\bmod m)}}^{\infty} \frac{\mu(d)}{d},
$$

which does not necessarily vanish. This means that the analogous constant $\left.\sigma_{1}(F, w, \mathbf{k m o d} m)\right)$ is brought into play, so that we cannot prove the corresponding weak approximation result without further information on $w, \mathbf{k}$ and $m$. Indeed for $n=3$ the analogue of Corollary 3 for primitive vectors is false. For example there is no primitive integer zero of $x_{1} x_{2}-x_{3}^{2}$ with $x_{1}, x_{2}, x_{3}>0$ and $\left.x_{1}, x_{2}, x_{3} \equiv \mathfrak{d} \bmod 3\right)$, even though there are such zeros in $\mathbb{R}$ and $\mathbb{Q}_{3}$.

\section{Smooth Weights}

Our analysis will make extensive use of 'smooth weight functions', and this section is therefore devoted to a discussion of their construction and their basic 
properties. We shall say that a function $w: \mathbb{R}^{n} \rightarrow \mathbb{C}$ is a 'smooth weight function,' or often just 'a weight function,' if it is an infinitely differentiable function of compact support. For such a function we shall define $n(w)$ to be the corresponding value of $n$, and $\operatorname{Rad}(w)$ to be the smallest $R$ such that $w$ is supported in the hypercube $[-R, R]^{n}$. Moreover, for every non-negative integer $j$ we define

$$
\kappa_{j}(w)=\max \left\{\left|\frac{\partial^{j_{1}+\ldots+j_{n}} w}{\partial^{j_{1}} x_{1} \ldots \partial^{j_{n}} x_{n}}\right|: \mathbf{x} \in \mathbb{R}^{n}, \sum j_{i}=j\right\} .
$$

Now, if $S$ is any collection of parameters, we shall define $\mathcal{C}(S)$ to be the set of those weight functions for which each of the numbers

$$
n(w), \operatorname{Rad}(w), \kappa_{0}(w), \kappa_{1}(w), \kappa_{2}(w), \ldots
$$

can be bounded by corresponding quantities

$$
n(S), \operatorname{Rad}(S), \kappa_{0}(S), \kappa_{1}(S), \kappa_{2}(S), \ldots
$$

depending only on parameters in the set $S$. In particular we shall put $\mathcal{C}=\mathcal{C}(\emptyset)$. By $\mathcal{C}^{+}(S)$, or $\mathcal{C}^{+}$we shall mean the corresponding set of weight functions whose values are non-negative real numbers. We note at once that $w \in \mathcal{C}(w)$, as already remarked in the introduction.

We begin by recording some elementary observations.

1. The function

$$
w_{0}(x)=\left\{\begin{array}{cl}
\exp \left(-\left(1-x^{2}\right)^{-1}\right), & |x|<1 \\
0, & |x| \geq 1
\end{array}\right.
$$

is in $\mathcal{C}^{+}$, and the function

$$
w_{0}^{(n)}(\mathbf{x})=\prod_{i=1}^{n} w_{0}\left(x_{i}\right), \quad \mathbf{x} \in \mathbb{R}^{n}
$$

is in $\mathcal{C}^{+}(n)$.

2. If $w_{1}$ and $w_{2}$ are in $\mathcal{C}(S)$, and $n\left(w_{1}\right)=n\left(w_{2}\right)$, then $w_{1}+w_{2}$ and $w_{1} w_{2}$ are also in $\mathcal{C}(S)$.

3. If $w \in \mathcal{C}(S)$ and $\lambda$ is any complex number satisfying $|\lambda| \leq \Lambda$, then

$$
\lambda w \in \mathcal{C}(S, \Lambda) .
$$

4. If $w \in \mathcal{C}(S)$, then for any $\mathbf{t} \in \mathbb{R}^{n(w)-1}$ the function

$$
w_{\mathbf{t}}(x)=w(x, \mathbf{t})
$$

is in $\mathcal{C}(S)$. 
5. Let $w \in \mathcal{C}(S)$. Let $M$ be a real square matrix of size $n(w)$, and suppose that the entries of both $M$ and $M^{-1}$ have modulus at most $K$. Then $w(M \mathbf{x}) \in$ $\mathcal{C}(S, K)$. Moreover $\operatorname{Rad}(w(M \mathbf{x})) \ll_{K, n(w)} \operatorname{Rad}(w(\mathbf{x}))$. In particular if $M$ is an orthogonal matrix, then $w(M \mathbf{x}) \in \mathcal{C}(S)$.

6. If $w \in \mathcal{C}(S)$ and $\mathbf{c} \in \mathbb{R}^{n(w)}$ satisfies $\|\mathbf{c}\| \leq K$, then $w(\mathbf{x}+\mathbf{c}) \in \mathcal{C}(S, K)$.

7. If $w \in \mathcal{C}(S)$, then the Fourier transform $\hat{w}$ is infinitely differentiable, and the $k$-th order partial derivatives are $O_{k, S, A}\left(\{1+\|\mathbf{x}\|\}^{-A}\right)$. (Here, as later, $\|\mathbf{x}\|=\max _{i}\left|x_{i}\right|$.) This follows by repeated integration by parts.

We shall use these properties without further comment.

We can approximate the characteristic function $\chi_{\mathcal{R}}(\mathbf{x})$ of a bounded set $\mathcal{R}$ by a weight function, as follows.

Lemma 1 Let $\mathcal{R}$ be a bounded subset of $\mathbb{R}^{n}$, and suppose that $\mathcal{R}$ has Jordan content $c(\mathcal{R})$. Then for any $\varepsilon>0$ there are weight functions $w_{ \pm}(\mathbf{x}) \in \mathcal{C}^{+}(\mathcal{R}, n, \varepsilon)$, such that

$$
w_{-} \leq \chi_{\mathcal{R}} \leq w_{+}
$$

and

$$
c(\mathcal{R})-\varepsilon \leq \int w_{-}(\mathbf{x}) d \mathbf{x} \leq c(\mathcal{R}) \leq \int w_{+}(\mathbf{x}) d \mathbf{x} \leq c(\mathcal{R})+\varepsilon .
$$

To construct the function $w_{-}$we begin by observing that, by definition of the Jordan content, there is a finite set of disjoint $n$-dimensional cuboids

$$
C_{1}, \ldots, C_{N} \subseteq \mathcal{R}
$$

whose edges are parallel to the coordinate axes, with total volume at least $c(\mathcal{R})-\varepsilon / 2$. It therefore suffices to find $w_{i} \in \mathcal{C}^{+}\left(C_{i}, n, \varepsilon\right)$, with $w_{i}(\mathbf{x}) \leq \chi_{C_{i}}$, for which

$$
\operatorname{vol}\left(C_{i}\right)-\varepsilon / 2 N \leq \int w_{i}(\mathbf{x}) d \mathbf{x}
$$

since we can then take $w_{-}=\sum_{i} w_{i}$. To do this we shall use the function $w_{0}$ given by (2.1). We set

$$
c_{0}=\int_{-\infty}^{\infty} w_{0}(x) d x
$$

and we write

$$
w(x ; A, B, \eta)=\eta^{-1} c_{0}^{-1} \int_{A+\eta}^{B-\eta} w_{0}\left(\frac{x-y}{\eta}\right) d y .
$$

Since

$$
\int_{A+\eta}^{B-\eta} w_{0}\left(\frac{x-y}{\eta}\right) d y \leq \int_{-\infty}^{\infty} w_{0}\left(\frac{x-y}{\eta}\right) d y=\eta c_{0},
$$

it follows that $0 \leq w(x ; A, B, \eta) \leq 1$ for all $x \in \mathbb{R}$. Moreover, since $w_{0}$ is supported in $[-1, \overline{1}]$, one sees that $w(x ; A, B, \eta)$ is supported in $[A, B]$. Finally 
we observe that

$$
\begin{aligned}
\int_{-\infty}^{\infty} w(x ; A, B, \eta) d x & =\eta^{-1} c_{0}^{-1} \int_{A+\eta}^{B-\eta} \int_{-\infty}^{\infty} w_{0}\left(\frac{x-y}{\eta}\right) d x d y \\
& =\eta^{-1} c_{0}^{-1} \int_{A+\eta}^{B-\eta} \eta c_{0} d y \\
& =B-A-2 \eta
\end{aligned}
$$

Hence if $C_{i}=\prod_{j}\left[A_{j}, B_{j}\right]$, we shall take

$$
w_{i}=\prod_{j=1}^{n} w\left(x_{j} ; a_{j}, B_{j}, \eta\right)
$$

and if $\eta$ is small enough the estimate (2.3) will hold. Of course one can construct the function $w_{+}(\mathbf{x})$ in a completely analogous way. This completes the proof of Lemma 1.

There are many situations in which one is integrating over a region which needs to be subdivided into smaller regions for some purpose or other. The following lemma provides the analogous process when one thinks of weight functions as corresponding to the characteristic function of the region concerned.

Lemma 2 Let $w \in \mathcal{C}(S)$ with $n(w)=n$. Then for any $\delta$ in the range $0<\delta \leq 1$ there is a function $w_{\delta}(\mathbf{x}, \mathbf{y}) \in \mathcal{C}(S)$ with $\mathbf{x}, \mathbf{y} \in \mathbb{R}^{n}$, such that

$$
w(\mathbf{x})=\delta^{-n} \int w_{\delta}\left(\frac{\mathbf{x}-\mathbf{y}}{\delta}, \mathbf{y}\right) d \mathbf{y} .
$$

Moreover $\operatorname{Rad}\left(w_{\delta}(*, \mathbf{y})\right) \leq 1$ for every $\mathbf{y}$, and the function

$$
F(\mathbf{x})=w_{\delta}\left(\delta^{-1}(\mathbf{x}-\mathbf{y}), \mathbf{y}\right)
$$

has $\operatorname{supp}(F) \subseteq \operatorname{supp}(w)$ for every $\mathbf{y}$.

In applications we will decompose a weighted integral

$$
\int w(\mathbf{x}) f(\mathbf{x}) d \mathbf{x}
$$

as a 'sum' $\int I(\mathbf{y}) d \mathbf{y}$ of integrals

$$
I(\mathbf{y})=\delta^{-n} \int w_{\delta}\left(\frac{\mathbf{x}-\mathbf{y}}{\delta}, \mathbf{y}\right) f(\mathbf{x}) d \mathbf{x}
$$

each of which is over a subregion of size at most $\delta$.

To prove the lemma we take $w_{\delta}(\mathbf{u}, \mathbf{v})=c_{0}^{-n} w_{0}^{(n)}(\mathbf{u}) w(\delta \mathbf{u}+\mathbf{v})$. Clearly this belongs to $\mathcal{C}(S)$, since $\delta \leq 1$. Moreover one trivially finds that

$$
\int w_{\delta}\left(\delta^{-1}(\mathbf{x}-\mathbf{y}), \mathbf{y}\right) d \mathbf{y}=w(\mathbf{x}) c_{0}^{-n} \int w_{0}^{(n)}\left(\frac{\mathbf{x}-\mathbf{y}}{\delta}\right) d \mathbf{y}=\delta^{n} w(\mathbf{x})
$$


as required. The remaining statements of the lemma are immediate consequences of our choice of the function $w_{\delta}$.

Although the product of weight functions is automatically another weight function, the situation with quotients is naturally a little more awkward. The following result will be of importance in this context.

Lemma 3 Let $w \in \mathcal{C}(S)$, and let positive real numbers $K_{1}, K_{2}, \ldots$ be given. Suppose that the complex valued function $g(\mathbf{x})$ is defined on $\operatorname{supp}(w)$ and satisfies $|g| \geq 1$ and

$$
\left|\frac{\partial^{j_{1}+\ldots+j_{n}} g(\mathbf{x})}{\partial^{j_{1}} x_{1} \ldots \partial^{j_{n}} x_{n}}\right| \leq K_{j} \quad\left(\sum j_{i}=j \geq 1\right)
$$

there. Then $g^{-1} w \in \mathcal{C}\left(S, K_{1}, K_{2}, \ldots\right)$.

Strictly speaking we should extend the definition of $g$, by taking $g(x)=1$, say, for $x \notin \operatorname{supp}(w)$, so that $g^{-1} w$ is defined on the whole of $\mathbb{R}^{n}$.

To prove the lemma it suffices to bound the partial derivatives of $g^{-1} w$ in terms of $S, K_{1}, K_{2} \ldots$ However one readily sees by induction on $j$, that any $j$-th order partial derivative of $g^{-1} w$ can be written as a sum of terms $g^{-k-1} P$ with $k \leq j$, where $P$ is a polynomial in various partial derivatives of $g$ and $w$. The required bound then follows.

\section{Proof of Theorems 1 and 2}

We begin by defining

$$
\omega(x)=4 c_{0}^{-1} w_{0}(4 x-3),
$$

where $w_{0}$ is the function given by (2.1), and $c_{0}$ is given by (2.4). Thus $\omega(x)$ is infinitely differentiable, supported in $(1 / 2,1)$, and satisfies

$$
0 \leq \omega(x) \ll 1
$$

and

$$
\int_{-\infty}^{\infty} \omega(x) d x=1
$$

Now if $Q>1$, the Poisson summation formula yields

$$
\sum_{q=1}^{\infty} \omega\left(\frac{q}{Q}\right)=\sum_{n} \int_{-\infty}^{\infty} \omega\left(\frac{x}{Q}\right) e(-n x) d x .
$$

The term $n=0$ produces a contribution $Q$, in view of (3.2), while non-zero values of $n$ produce contributions $O_{N}\left(Q(Q|n|)^{-N}\right)$, on integrating by parts $N$ times. We may therefore define $c_{Q}$ by writing

$$
\sum_{q=1}^{\infty} \omega\left(\frac{q}{Q}\right)=c_{Q}^{-1} Q
$$


so that $c_{Q}=1+O_{N}\left(Q^{-N}\right)$, as required.

Now if $n$ is a positive integer, then as $q$ runs over divisors of $n$, so does $n / q$. It therefore follows that

$$
\sum_{q \mid n}\left\{\omega\left(\frac{q}{Q}\right)-\omega\left(\frac{n / q}{Q}\right)\right\}=0 .
$$

Similarly if $n$ is strictly negative we will have

$$
\sum_{q \mid n}\left\{\omega\left(\frac{q}{Q}\right)-\omega\left(\frac{|n| / q}{Q}\right)\right\}=0 .
$$

On the other hand, if $n=0$ then $\omega(|n| /(q Q))=0$, since $\omega(0)=0$. Moreover

$$
\sum_{q \mid n} \omega(q / Q)=\sum_{q=1}^{\infty} \omega(q / Q)=c_{Q}^{-1} Q .
$$

It follows that

$$
c_{Q} Q^{-1} \sum_{q \mid n}\left\{\omega\left(\frac{q}{Q}\right)-\omega\left(\frac{|n|}{q Q}\right)\right\}= \begin{cases}1, & n=0 \\ 0, & n \neq 0 .\end{cases}
$$

In order to put the sum on the left into a form more readily comparable with the corresponding formula arising from the classical circle method, we pick out the condition $q \mid n$ using exponential sums. This yields

$$
\sum_{q \mid n}\left\{\omega\left(\frac{q}{Q}\right)-\omega\left(\frac{|n|}{q Q}\right)\right\}=\sum_{q=1}^{\infty} \frac{1}{q} \sum_{a(\bmod q)} e_{q}(a n)\left\{\omega\left(\frac{q}{Q}\right)-\omega\left(\frac{|n|}{q Q}\right)\right\} .
$$

Now, to put the fractions $a / q$ into lowest terms we set $(a, q)=j, a=j b$, and $q=j l$, so that

$$
\begin{aligned}
& \sum_{q=1}^{\infty} \frac{1}{q} \sum_{a(\bmod q)} e_{q}(\text { an })\left\{\omega\left(\frac{q}{Q}\right)-\omega\left(\frac{|n|}{q Q}\right)\right\} \\
&=Q^{-1} \sum_{l=1}^{\infty} \sum_{b(\bmod l)}{ }^{*} e_{l}(b n) h\left(Q^{-1} l, Q^{-2} n\right),
\end{aligned}
$$

where

$$
h(x, y)=\sum_{j} \frac{1}{x j}\{\omega(x j)-\omega(|y| / x j)\} .
$$

After renaming the variables we now have

$$
\delta_{n}=c_{Q} Q^{-2} \sum_{q=1}^{\infty} \sum_{a(\bmod q)}^{*} e_{q}(a n) h\left(Q^{-1} q, Q^{-2} n\right),
$$


as required for Theorem 1. Moreover, since $\omega$ is supported in $\left[\frac{1}{2}, 1\right]$ we see that

$$
h(x, y) \ll\left\{\sum_{1 / 2 x \leq j \leq 1 / x}+\sum_{|y| / x \leq j \leq 2|y| / x}\right\}(x j)^{-1} \ll x^{-1},
$$

as claimed in the theorem. It remains to check that the functions

$$
h_{1}(x, y)=\sum_{j} \frac{1}{x j} \omega(x j)
$$

and

$$
h_{2}(x, y)=\sum_{j} \frac{1}{x j} \omega(|y| / x j)
$$

are both infinitely differentiable for $(x, y) \in(0, \infty) \times \mathbb{R}$. However if $\left(x_{0}, y_{0}\right)$ is any such point, and $(x, y) \in\left(x_{0} / 2,2 x_{0}\right) \times\left(y_{0}-1, y_{0}+1\right)$, the two sums in question reduce to finite sums with

$$
\frac{1}{4 x_{0}} \leq j \leq \frac{2}{x_{0}}
$$

and

$$
\frac{\left|y_{0}\right|-1}{2 x_{0}} \leq j \leq \frac{4\left(\left|y_{0}\right|+1\right)}{x_{0}}
$$

respectively. The result then follows, since the individual summands are infinitely differentiable.

We turn now to Theorem 2. The first statement of the theorem follows immediately from Theorem 1, via the observation that

$$
N^{(0)}(F, w)=\sum w(\mathbf{x}) \delta_{F(\mathbf{x})} .
$$

To prove the second assertion we split the values of $\mathbf{x}$ into their respective residue classes $\mathbf{b} \bmod q$ ), so that

$$
\sum_{\mathbf{x}} w(\mathbf{x}) e_{q}(a F(\mathbf{x})) h\left(Q^{-1} q, Q^{-2} F(\mathbf{x})\right)=\sum_{\mathbf{b}(\bmod q)} e_{q}(a F(\mathbf{b})) \sum_{\mathbf{y}} f(\mathbf{y}),
$$

where we have written, temporarily,

$$
f(\mathbf{y})=w(\mathbf{b}+q \mathbf{y}) h\left(Q^{-1} q, Q^{-2} F(\mathbf{b}+q \mathbf{y})\right) .
$$

The $n$-dimensional Poisson summation formula now yields

$$
\sum_{\mathbf{y}} f(\mathbf{y})=\sum_{\mathbf{c}} \hat{f}(\mathbf{c})
$$

with

$$
\hat{f}(\mathbf{c})=\int_{\mathbb{R}^{n}} f(\mathbf{y}) e(-\mathbf{c} \cdot \mathbf{y}) d \mathbf{y} .
$$


This holds indeed for any smooth compactly supported function $f$. If we now substitute $\mathbf{x}=\mathbf{b}+q \mathbf{y}$ we find that

$$
\hat{f}(\mathbf{c})=q^{-n} e_{q}(\mathbf{b . c}) I_{q}^{(0)}(\mathbf{c}),
$$

where

$$
I_{q}^{(0)}(\mathbf{c})=\int_{\mathbb{R}^{n}} w(\mathbf{x}) h\left(Q^{-1} q, Q^{-2} F(\mathbf{x})\right) e_{q}(-\mathbf{c} \cdot \mathbf{x}) d \mathbf{x},
$$

as in the statement of Theorem 2. The result now follows on rearranging the summations over $q, \mathbf{c}$ and $a$.

\section{The Function $h(x, y)$}

In this section we shall establish the basic properties of the function $h(x, y)$. It has already been observed that $h(x, y) \ll x^{-1}$. We shall first prove a more general result.

Lemma 4 The function $h(x, y)$ vanishes when $x \geq 1$ and $|y| \leq x / 2$. When $x \leq 1$ and $|y| \leq x / 2$ the function $h(x, y)$ is constant with respect to $y$, taking the value

$$
\sum_{j=1}^{\infty}(x j)^{-1} \omega(x j) \ll x^{-1}
$$

Moreover

$$
\frac{\partial^{m} h(x, y)}{\partial x^{m}} \ll_{m} x^{-m-1}, \quad(x \leq 1, \quad|y| \leq x / 2) .
$$

When $|y| \geq x / 2$ we have

$$
\frac{\partial^{m+n} h(x, y)}{\partial x^{m} \partial y^{n}} \ll_{m, n} x^{-m-1}|y|^{-n} .
$$

The first statement of the lemma is immediate, since $\omega$ is supported in $(1 / 2,1)$ and

$$
|y| / x j \leq 1 / 2, \quad x j \geq 1
$$

for $j \geq 1$. The second statement follows similarly. Moreover

$$
\frac{\partial^{t} \omega(x j)}{\partial x^{t}}=j^{t} \omega^{(t)}(x j) \ll_{t} x^{-t}
$$

for $1 / 2<x j<1$. Hence

$$
\frac{\partial^{m} x^{-1} \omega(x j)}{\partial x^{m}} \ll_{m} x^{-m-1}
$$

by Leibnitz' formula. The third assertion of the lemma then follows.

Turning to the final part of the lemma we observe that the terms $(x j)^{-1} \omega(x j)$ in the definition of $h(x, y)$ only contribute to the partial derivative when $x \leq 1$ 
and $n=0$, in which case they produce $O_{m}\left(x^{-m-1}\right)$ as before. This is satisfactory. For the other terms we observe that

$$
\frac{\partial^{m+n} x^{-1} \omega(y / x j)}{\partial x^{m} \partial y^{n}}=y^{-n} x^{-m-1} \sum_{0 \leq t \leq m} c_{m, n, t}\left(\frac{y}{x j}\right)^{n+t} \omega^{(n+t)}\left(\frac{y}{x j}\right),
$$

for certain constants $c_{m, n, t}$, as a simple induction argument shows. We may then see that

$$
\frac{\partial^{m+n} x^{-1} \omega(y / x j)}{\partial x^{m} \partial y^{n}} \ll_{m, n} x^{-m-1}|y|^{-n},
$$

since

$$
\left(\frac{y}{x j}\right)^{n+t} \omega^{(n+t)}\left(\frac{y}{x j}\right) \ll_{n, t} 1 .
$$

The required result now follows, using the fact that $\sum j^{-1} \ll 1$ if we sum only for those $j$ for which $\omega(x j)$ and $\omega(|y| / x j)$ are non-vanishing.

Our second result shows that the sum involved in $h(x, y)$ nearly cancels if $x=o(\min \{1,|y|\})$.

Lemma 5 Let $N, m$ and $n$ be non-negative integers. Then

$$
\frac{\partial^{m+n} h(x, y)}{\partial x^{m} \partial y^{n}} \ll_{N, m, n} x^{-1-m-n}\left(x^{N}+\min \left\{1,(x /|y|)^{N}\right\}\right) .
$$

The term $x^{N}$ on the right may be omitted for $n \neq 0$.

This estimate is an immediate corollary of Lemma 4 unless $x \leq|y|$, as we now assume. Moreover, when $n=0$ and $x \geq 1$, the result also follows from Lemma 4. We shall therefore suppose that $x \leq 1$ if $n=0$.

For the proof of Lemma 5 we shall use the Euler-Maclaurin summation formula. This takes the form

$$
\begin{aligned}
\sum_{a<n \leq b} f(n)=\int_{a}^{b} f(t) d t & \left(a-[a]-\frac{1}{2}\right) f(a)-\left(b-[b]-\frac{1}{2}\right) f(b) \\
+\sum_{k=2}^{N} \frac{(-1)^{k}}{k !}\left[P_{k}(t) f^{(k-1)}(t)\right]_{a}^{b} & -\frac{(-1)^{N}}{N !} \int_{a}^{b} P_{N}(t) f^{(N)}(t) d t
\end{aligned}
$$

for any infinitely differentiable function $f$ on $[a, b]$. Here $P_{k}(t)$ denotes the $k$-th periodic Bernoulli function.

We apply this to the sums

$$
\sum_{j}(x j)^{-1} \omega(x j)
$$

and

$$
\sum_{j}(x j)^{-1} \omega(|y| / x j)
$$


observing that the functions $f(t)=(x t)^{-1} \omega(x t)$ and $f(t)=(x t)^{-1} \omega(|y| / x t)$ are supported on $(1 / 2 x, 1 / x)$ and $(|y| / x, 2|y| / x)$ respectively. Thus

$$
\begin{aligned}
\sum_{j}(x j)^{-1} \omega(x j)= & \int_{1 / 2 x}^{1 / x}(x t)^{-1} \omega(x t) d t \\
& \quad-\frac{(-1)^{N}}{N !} \int_{1 / 2 x}^{1 / x} P_{N}(t) \frac{\partial^{N}}{\partial t^{N}}(x t)^{-1} \omega(x t) d t,
\end{aligned}
$$

and

$$
\begin{aligned}
\sum_{j}(x j)^{-1} \omega(|y| / x j)= & \int_{|y| / x}^{2|y| / x} \\
& (x t)^{-1} \omega(|y| / x t) d t \\
& \quad-\frac{(-1)^{N}}{N !} \int_{|y| / x}^{2|y| / x} P_{N}(t) \frac{\partial^{N}}{\partial t^{N}}(x t)^{-1} \omega(|y| / x t) d t .
\end{aligned}
$$

We may regard these expressions as being a main term plus an error term. The two main terms agree, as one sees by the substitution $u=|y| /\left(x^{2} t\right)$. Thus, if we set $w(u)=u^{-1} \omega(u)$, the two error terms become

$$
-x^{N-1} \frac{(-1)^{N}}{N !} \int_{1 / 2}^{1} P_{N}(u / x) w^{(N)}(u) d u
$$

and

$$
-\frac{1}{x}\left(\frac{x}{|y|}\right)^{N} \frac{(-1)^{N}}{N !} \int_{1}^{2} P_{N}(u|y| / x) \frac{d^{N}}{d u^{N}} u^{-2} w\left(u^{-1}\right) d u
$$

on substituting $t=u / x$ and $t=u|y| / x$ respectively. When we differentiate with respect to $x$ and $y$ we introduce factors of the form

$$
x^{-m}\left(\frac{u}{x}\right)^{j}, \quad(j \leq m, n=0)
$$

and

$$
x^{-m}|y|^{-n}\left(\frac{u|y|}{x}\right)^{k}, \quad(k \leq m+n)
$$

respectively, and we replace the function $P_{N}$ by one of its derivatives. Since the functions $w(u)$ and $u^{-2} w\left(u^{-1}\right)$ are both in the class $\mathcal{C}$, the error terms therefore contribute $O_{N}\left(x^{N-m-1}\left\{1+x^{-m}\right\}\right)$ and $O_{N}\left(x^{-m-1}|y|^{-n}(|y| / x)^{m+n-N}\right)$, respectively. The former of these is present only for $n=0$, in which case we have $x \leq 1$, be our initial assumption. The first error term may therefore be replaced by $O_{N}\left(x^{-1-2 m+N}\right)$. Since $x \leq|y|$ the error terms then suffice for the lemma, on redefining $N$.

Our third lemma describes the average behaviour of $h(x, y)$ for small $y$. It turns out that this result is the key to the derivation of the 'singular integral' in our form of the circle method. One curious fact is that the constant 1 on the right hand side below is independent of the precise form of the weight function $w_{0}(x)$ used in the construction of $\omega$. Any other infinitely differentiable function 
supported in $[-1,1]$ could have been used instead. Of course, this is inevitable, since the counting function $N(F, w)$ in Theorem 2 is independent of the choice of $w_{0}$.

Lemma 6 Suppose that $x \ll \min (1, X)$. Then

$$
\int_{-X}^{X} h(x, y) d y=1+O_{N}\left(X x^{N-1}\right)+O_{N}\left(\left(\frac{X}{x}\right)^{-N}\right)
$$

for any $N>0$.

For the proof we begin by handling the sum $\sum(x j)^{-1} \omega(x j)$ as in the proof of Lemma 5. This yields

$$
\sum(x j)^{-1} \omega(x j)=x^{-1} \int_{0}^{\infty} \omega(u) \frac{d u}{u}+O_{N}\left(x^{N-1}\right) .
$$

When we integrate for $-X \leq y \leq X$ the above error term is satisfactory for Lemma 6 . It therefore remains to show that

$$
\frac{2 X}{x} \int_{0}^{\infty} \omega(u) \frac{d u}{u}-2 \sum_{j} \frac{1}{x j} \int_{0}^{X} \omega(y / x j) d y=1+O_{N}\left(\left(\frac{X}{x}\right)^{-N}\right) .
$$

The leading term on the right arises from our assumption (3.2) that

$$
\int_{-\infty}^{\infty} \omega(x) d x=1
$$

On substituting $Y=X / x$ we therefore see that it suffices to prove the following result.

Lemma 7 If $w(u) \in \mathcal{C}$ is supported in $[1 / 2,1]$ then

$$
Y \int_{0}^{\infty} w(u) \frac{d u}{u}-\sum_{j} \int_{0}^{Y / j} w(u) d u=\frac{1}{2} \int_{0}^{\infty} w(u) d u+O_{N}\left(Y^{-N}\right)
$$

for any $N>0$.

To prove Lemma 7 we begin by setting

$$
\phi(t)=\int_{0}^{Y / t} w(u) d u
$$

so that $\phi(t)=0$ for $t \geq 2 Y$, and

$$
\phi(t)=\int_{0}^{\infty} w(u) d u, \quad(t \leq Y) .
$$

Moreover

$$
\phi^{\prime}(t)=-Y t^{-2} w(Y / t)
$$


is supported in $[Y, 2 Y]$ and has derivatives of all orders, satisfying

$$
\phi^{(k)}(t) \ll_{k} Y^{-k}, \quad(Y \leq t \leq 2 Y) .
$$

We now apply the Euler-Maclaurin summation formula (4.1), taking $a \rightarrow 0$ and $b>2 Y$. This yields

$$
\sum_{j=1}^{\infty} \phi(j)=\int_{0}^{\infty} \phi(x) d x-\frac{1}{2} \int_{0}^{\infty} w(u) d u-\frac{(-1)^{N}}{N !} \int_{Y}^{2 Y} P_{N}(t) \phi^{(N)}(t) d t .
$$

However the first integral on the right is

$$
\int_{0}^{2 Y} \int_{1 / 2}^{Y / t} w(u) d u d t=\int_{1 / 2}^{1} w(u) \int_{0}^{Y / u} d t d u=Y \int_{1 / 2}^{1} w(u) \frac{d u}{u},
$$

while the third integral is $O_{N}\left(Y^{1-N}\right)$, in view of (4.2). Lemma 7, and hence Lemma 6, now follows.

As a companion to Lemma 6 we will need the following bound.

Lemma 8 Let $n$ be a positive integer and suppose that $x \ll \min (1, X)$. Then

$$
\int_{-X}^{X} y^{n} h(x, y) d y \ll_{N} X^{n}\left(X x^{N-1}+\left(\frac{X}{x}\right)^{-N}\right)
$$

for any $N>0$.

This is trivial for odd values of $n$, since $h(x, y)$ is an even function of $y$, and the integral vanishes. We shall therefore take $n \geq 2$ to be even and examine

$$
\int_{0}^{X} y^{n} h(x, y) d y \text {. }
$$

We begin our argument as in the proof of Lemma 6 , showing that

$$
\begin{array}{r}
\int_{0}^{X} y^{n} \sum_{j}(x j)^{-1} \omega(x j) d y=\int_{0}^{X} y^{n} x^{-1} \int_{0}^{\infty} \omega(u) \frac{d u}{u} d y \\
+O_{N}\left(X^{n+1} x^{N-1}\right) .
\end{array}
$$

We evaluate

$$
\sum_{j}(x j)^{-1} \omega(|y| / x j)
$$

by the Euler-Maclaurin summation formula, as in the proof of Lemma 5, to give

$$
x^{-1} \int_{0}^{\infty} \omega(u) \frac{d u}{u}-\frac{1}{x}\left(\frac{x}{y}\right)^{n} \frac{(-1)^{n}}{n !} \int_{1}^{2} P_{n}(u y / x) \frac{d^{n}}{d u^{n}} u^{-2} w\left(u^{-1}\right) d u .
$$

Here the first term produces a contribution which cancels with the double integral in (4.3), and it therefore suffices to show that

$$
\int_{0}^{X} \int_{1}^{2} P_{n}(u y / x) w(u) d u d y \ll_{n, N} X(x / X)^{N+1-n}
$$


for any function $w(u) \in \mathcal{C}$ supported in $[1,2]$.

To establish (4.4) we substitute $y=z x / u$ and perform the integration over $z$, using the fact that

$$
\int_{0}^{Z} P_{n}(z) d z=\frac{P_{n+1}(Z)}{n+1}-\frac{P_{n+1}(0)}{n+1}=\frac{P_{n+1}(Z)}{n+1} .
$$

Here our assumption that $n$ is even and strictly positive plays a crucial rôle. We now see that the left hand side of (4.4) is

$$
\frac{x}{n+1} \int_{1}^{2} P_{n+1}(u X / x) w(u) \frac{d u}{u},
$$

and repeated integration by parts yields

$$
(-1)^{N-n} \frac{n !}{(N+1) !}\left(\frac{x}{X}\right)^{N-n} x \int_{1}^{2} P_{N+1}(u X / x) \frac{d^{N-n}}{d u^{N-n}}\left(u^{-1} w(u)\right) d u,
$$

A trivial estimate then yields (4.4), and hence Lemma 8.

Combining our various estimates we can now show that for small values of $x$ the function $h(x, y)$ acts very much like a delta-function.

Lemma 9 Let $f \in \mathcal{C}(S)$. Then if $x \ll 1$ we have

$$
\int f(y) h(x, y) d y=f(0)+O_{S, M}\left(x^{M}\right)
$$

for any $M>0$.

We write $X=\min \left\{1, x^{1 / 2}\right\}$ and apply Lemma 5 to show that $h(x, y) \ll x^{N / 2-1}$ for $|y| \geq X$, for any $N>0$. Since $f \ll_{S} 1$, the range $|y| \geq X$ therefore makes a satisfactory contribution. When $|y| \leq X$ we may expand $f(y)$ in a Taylor series, producing an approximating polynomial of degree $2 M$ together with an error $O_{S, M}\left(X^{2 M+1}\right)$. Since $h(x, y) \ll x^{-1}$, by Lemma 4, this latter error contributes a total $\ll_{S, M} x^{-1} X^{2 M+2}$, which is sufficient. Finally, by Lemmas 6 and 8 , the approximating polynomial produces a main term $f(0)$, together with error terms $O_{S, N}\left(x^{N-1}\right)$ and $O_{S, N}\left(x^{N} X^{-N}\right)$. These too are satisfactory for $N=2 M$.

\section{$5 \quad$ Weighted Exponential Integrals}

In this section we shall study multiple integrals of the form

$$
\int w(\mathbf{x}) e(f(\mathbf{x})) d \mathbf{x}
$$

where $w(\mathbf{x})$ is a weight function in $\mathcal{C}(S)$ and $f(\mathbf{x})$ is a suitably smooth real valued function. In the literature there are rather few investigations of multiple exponential integrals in 3 or more dimensions. Even in two dimensions the 
results are encumbered with unpleasant side conditions. These arise because unweighted integrals are used, the integral being over an appropriate subset $\mathcal{D}$ of Euclidean space. Typical conditions required of $\mathcal{D}$ are those given by Srinivasan [18; page 285], who, wishing to consider sums and integrals over a region $D$, writes:

'We suppose that $D$ is a finite region in a $p$-dimensional Euclidean space and that any line parallel to any of the coordinate axes meets it in $O(1)$ straight line segments, and the same is true for the intersection of $D$ with regions of the type $f_{x_{i}} \leq$ const and $f_{x_{i}} \geq$ const, $i=1, \ldots, p$, where $f\left(x_{1}, \ldots, x_{p}\right)$ is a real function defined over $D$ such that the transformation $y_{i}=f_{x_{i}}, i=1, \ldots, p$ is one-one over $D$. We suppose further that any line parallel to any of the coordinate axes meets the surface got by equating to zero any of the second order partial derivatives of $f$ in $O(1)$ points.'

One aesthetic drawback here is that the conditions imposed are not invariant under linear changes of variable. All these difficulties are overcome when one uses weighted integrals. One can think of $\mathcal{D}$ as corresponding to $\operatorname{supp}(w)$ if one so wishes.

The estimate we shall prove corresponds to the 'first derivative' bound, see Titchmarsh [19; Lemmas 4.2 and 4.3], for example.

Lemma 10 Let $w(\mathbf{x}) \in \mathcal{C}(S)$ and let $f(\mathbf{x})$ be an infinitely differentiable real valued function defined on $\operatorname{supp}(w)$. Suppose that there is a positive real number $\lambda$, and a set $A=\left\{A_{2}, A_{3}, A_{4}, \ldots\right\}$ of positive real numbers such that, for all $\mathbf{x} \in \operatorname{supp}(w)$ we have

$$
|\nabla f| \geq \lambda
$$

and

$$
\left|\frac{\partial^{j_{1}+\ldots+j_{n}} f(\mathbf{x})}{\partial^{j_{1}} x_{1} \ldots \partial^{j_{n}} x_{n}}\right| \leq A_{j} \lambda, \quad\left(j=j_{1}+\ldots+j_{n} \geq 2\right) .
$$

Then for any $N>0$ we have

$$
\int w(\mathbf{x}) e(f(\mathbf{x})) d \mathbf{x} \ll_{N, S, A} \lambda^{-N}
$$

A number of remarks should be made here. In the first place, for the result to be of use we will require $\lambda \gg 1$. However if $\lambda$ is large enough we can achieve an arbitrary degree of precision, just by choosing $N$ appropriately. In contrast the corresponding result in Titchmarsh would give a bound $O\left(\lambda^{-1}\right)$ only. However our improved estimate is obtained at the cost of requiring $f$ to be infinitely differentiable, whereas Titchmarsh only needs $f$ to be once differentiable. We should also point out that in the one dimensional case there is a monotonicity condition on $f^{\prime}$. There is no natural analogue of this that one can use in higher dimensions. Instead our hypotheses ensure that none of the partial derivatives of $f$ can fluctuate too wildly. 
We shall prove the lemma by induction on $N$. We take as our induction hypothesis the statement that

$$
\int w(\mathbf{x}) e(f(\mathbf{x})) d \mathbf{x} \ll_{N, S, A} \lambda^{-N}
$$

for all $w \in \mathcal{C}(S, A)$. As the induction proceeds the dimension $n(w)$ will remain constant, but the function $w$ itself will vary, as will $\operatorname{supp}(w)$. When $N=0$ the required result is an immediate consequence of the bounds on $w$ and on $\operatorname{supp}(w)$ contained in the definition of the class $\mathcal{C}(S, A)$.

We begin the induction step by using Lemma 2 to produce integrals over smaller regions on which we can ensure not merely that $|\nabla f| \gg \lambda$, but that $\left|\partial f / \partial x_{j}\right| \gg \lambda$ for some index $j$. If $n=n(w)$ we shall choose $\delta=\left(1+2 n A_{2}\right)^{-1}$, whence

$$
\int w(\mathbf{x}) e(f(\mathbf{x})) d \mathbf{x}=\delta^{-n} \iint w_{\delta}\left(\frac{\mathbf{x}-\mathbf{y}}{\delta}, \mathbf{y}\right) e(f(\mathbf{x})) d \mathbf{x} d \mathbf{y} .
$$

Since $w_{\delta}(\mathbf{x}, \mathbf{y}) \in \mathcal{C}(S)$ we deduce that

$$
\int w(\mathbf{x}) e(f(\mathbf{x})) d \mathbf{x} \ll_{S, A} \int w_{\delta}\left(\frac{\mathbf{x}-\mathbf{y}}{\delta}, \mathbf{y}\right) e(f(\mathbf{x})) d \mathbf{x}
$$

for some $\mathbf{y}$. We shall write $w_{1}(\mathbf{x})=w_{\delta}\left(\delta^{-1}(\mathbf{x}-\mathbf{y}), \mathbf{y}\right)$ for short, and we note that, by Lemma $2, w_{1} \in \mathcal{C}(S, A)$, independently of $\mathbf{y}$. Since $|\nabla f(\mathbf{y})| \geq \lambda$ it follows that there is some index $j$ for which $\left|\partial f(\mathbf{y}) / \partial y_{j}\right| \geq \lambda / n$. Without loss of generality we may take $j=1$. The hypotheses of Lemma 10 now show that $\left|\partial^{2} f(\mathbf{x}) / \partial x_{1}^{2}\right| \leq A_{2} \lambda$. Our choice of $\delta$ therefore ensures that

$$
\left|\partial f(\mathbf{x}) / \partial x_{1}\right| \geq \lambda / 2 n
$$

whenever $\left|x_{1}-y_{1}\right| \leq \delta$, and, in particular, whenever

$$
w_{1}(\mathbf{x})=w_{\delta}\left(\delta^{-1}(\mathbf{x}-\mathbf{y}), \mathbf{y}\right) \neq 0 .
$$

We can now conclude that it suffices to establish the bound

$$
\int w_{1}(\mathbf{x}) e(f(\mathbf{x})) d \mathbf{x} \ll_{N, S, A} \lambda^{-N-1},
$$

where now (5.2) holds on $\operatorname{supp}\left(w_{1}\right)$.

To achieve this we shall write the integrand as

$$
\frac{w_{1}(\mathbf{x})}{2 \pi i f_{1}(\mathbf{x})} \frac{\partial}{\partial x_{1}} e(f(\mathbf{x}))
$$

where $f_{1}=\partial f / \partial x_{1}$. We can now integrate by parts with respect to $x_{1}$ to obtain

$$
\int w_{1}(\mathbf{x}) e(f(\mathbf{x})) d \mathbf{x}=-(\lambda / 2 n)^{-1} \int w_{2}(\mathbf{x}) e(f(\mathbf{x})) d \mathbf{x}
$$


where

$$
w_{2}(\mathbf{x})=\frac{\partial}{\partial x_{1}} \frac{w_{1}(\mathbf{x})}{2 \pi i(\lambda / 2 n)^{-1} f_{1}(\mathbf{x})} .
$$

According to (5.1) and (5.2) the function $g=(\lambda / 2 n)^{-1} f_{1}$ satisfies the conditions of Lemma 3 , with $K_{j}=2 n A_{j-1}$, so that

$$
\frac{w_{1}(\mathbf{x})}{2 \pi i(\lambda / 2 n)^{-1} f_{1}(\mathbf{x})}
$$

must be in $\mathcal{C}(S, A)$. It follows that $w_{2}(\mathbf{x})$ is also in $\mathcal{C}(S, A)$, so that our induction hypothesis yields the bound

$$
\int w_{2}(\mathbf{x}) e(f(\mathbf{x})) d \mathbf{x} \ll_{N, S, A} \lambda^{-N} .
$$

The required estimate (5.3) now follows, in view of (5.4). This completes the proof of Lemma 10.

\section{The Singular Integral}

We begin this section by specifying the class $\mathcal{C}_{0}(S)$ of functions for which the singular integral is most readily evaluated. Recall that $S$ is a list of parameters, amongst which is the function $G$. We take $\mathcal{C}_{0}(S)$ to be the set of weight functions $w \in \mathcal{C}(S)$ such that there exists a real number $R \ll_{S} 1$ with the property that, whenever $\left(x_{0}, \mathbf{y}\right) \in \operatorname{supp}(w)$, the function $G(x, \mathbf{y})$ satisfies

$$
\frac{\partial G(x, \mathbf{y})}{\partial x} \gg_{S} 1
$$

in the range $\left|x-x_{0}\right| \leq R$, and has exactly one zero there. For functions in $\mathcal{C}_{0}(S)$ we shall refer to the corresponding value of $x_{1}$ as being that which is determined by $\left(x_{2}, \ldots, x_{n}\right)$ via the equation $G(\mathbf{x})=0$. Of course if $x_{1}-x_{0}$ is not restricted to the range $[-R, R]$ specified above, there may be more than one solution. Equally, it may happen that the value of $x_{1}-x_{0}$ does lie in $[-R, R]$, but that the resulting vector $\mathbf{x}$ is not in $\operatorname{supp}(w)$. We observe that, for given $G$, whether or not a function $w \in \mathcal{C}(S)$ belongs to $\mathcal{C}_{0}(S)$ depends solely on properties of $\operatorname{supp}(w)$.

We proceed to prove Theorem 3 for weights in $\mathcal{C}_{0}(S)$. Indeed we shall establish the following uniform estimate.

Lemma 11 Let $w \in \mathcal{C}_{0}(S)$, for some set $S$, and set

$$
\sigma_{\infty}=\int \ldots \int \frac{w\left(x_{1}, \mathbf{y}\right)}{\partial G / \partial x_{1}} d \mathbf{y}
$$

where $x_{1}$ is determined by the condition $G(\mathbf{x})=0$, and $\mathbf{y}$ runs over all vectors in $\mathbb{R}^{n-1}$ for which there is at least one $x$ with $(x, \mathbf{y}) \in \operatorname{supp}(w)$. Then

$$
\frac{1}{2 \varepsilon} \int_{|G(\mathbf{x})| \leq \varepsilon} w(\mathbf{x}) d \mathbf{x}=\sigma_{\infty}+O_{S}(\varepsilon) .
$$


If, further, $w$ is real-valued and non-negative everywhere, and takes a strictly positive value for some real solution $\mathbf{x}$ of $G(\mathbf{x})=0$, then $\sigma_{\infty}>0$.

For the proof we shall write a typical vector $\mathbf{x} \in \operatorname{supp}(w)$ as $(x, \mathbf{y})$, and for such a $\mathbf{y}$ we take $x_{1}$ to be the unique solution of $G\left(x_{1}, \mathbf{y}\right)=0$ given in the definition of $\mathcal{C}(S)$. Then if $|G(\mathbf{x})| \leq \varepsilon$, we will have $\left|G(x, \mathbf{y})-G\left(x_{1}, \mathbf{y}\right)\right| \leq \varepsilon$, whence $\left|x-x_{1}\right| \ll_{S} \varepsilon$, in view of (6.1). It follows that $w(\mathbf{x})=w\left(x_{1}, \mathbf{y}\right)+O_{S}(\varepsilon)$. Thus

$$
\frac{1}{2 \varepsilon} \int_{|G(\mathbf{x})| \leq \varepsilon} w(\mathbf{x}) d \mathbf{x}=\frac{1}{2 \varepsilon} \int_{|G(\mathbf{x})| \leq \varepsilon} w\left(x_{1}, \mathbf{y}\right) d \mathbf{x}+O_{S}\left(\int_{|G(\mathbf{x})| \leq \varepsilon} d \mathbf{x}\right) .
$$

Here all three integrals are over subsets of $\operatorname{supp}(w)$. In the two integrals on the right, for each $\mathbf{y}=\left(x_{2}, \ldots, x_{n}\right)$ the available $x$ belong to an interval of length $\varepsilon$. In particular it follows that the error term is $O_{S}(\varepsilon)$. Moreover, for the first integral on the right we see by Taylor's Theorem that

$$
G(\mathbf{x})=\left(x-x_{1}\right) \frac{\partial G\left(x_{1}, \mathbf{y}\right)}{\partial x_{1}}+O_{S}\left(\varepsilon^{2}\right),
$$

since the second partial derivatives of $G$ will be $O_{S}(1)$ in the relevent region. It follows that for each $\mathbf{y}$ the condition $|G(x, \mathbf{y})| \leq \varepsilon$ prescribes an interval $I_{\mathbf{y}}$ for $x$, of length

$$
\frac{2 \varepsilon}{\partial G\left(x_{1}, \mathbf{y}\right) / \partial x_{1}}+O_{S}\left(\varepsilon^{2}\right) .
$$

Here again we have used the bound (6.1). If $\mathbf{y}$ is fixed, and $(x, \mathbf{y}) \in \operatorname{supp}(w)$ for all $x \in I_{\mathbf{y}}$, then

$$
\frac{1}{2 \varepsilon} \int_{|G(\mathbf{x})| \leq \varepsilon} w\left(x_{1}, \mathbf{y}\right) d x=\frac{w\left(x_{1}, \mathbf{y}\right)}{\partial G / \partial x_{1}}+O_{S}(\varepsilon),
$$

the integral on the left being over those $x$ for which $(x, \mathbf{y}) \in \operatorname{supp}(w)$. On the other hand, if $\left(x^{*}, \mathbf{y}\right) \notin \operatorname{supp}(w)$ for some $x^{*}$ in $I_{\mathbf{y}}$, then $w\left(x^{*}, \mathbf{y}\right)=0$ and $x_{1}=x^{*}+O_{S}(\varepsilon)$, whence $w\left(x_{1}, \mathbf{y}\right) \ll_{S} \varepsilon$. It then follows that

$$
\frac{1}{2 \varepsilon} \int_{|G(\mathbf{x})| \leq \varepsilon} w\left(x_{1}, \mathbf{y}\right) d x \ll_{S} \operatorname{meas}\{x:|G(x, \mathbf{y})| \leq \varepsilon\} \ll_{S} \varepsilon,
$$

and

$$
\frac{w\left(x_{1}, \mathbf{y}\right)}{\partial G / \partial x_{1}} \ll_{S} \varepsilon
$$

so that (6.2) still holds.

We therefore deduce that

$$
\frac{1}{2 \varepsilon} \int_{|G(\mathbf{x})| \leq \varepsilon} w(\mathbf{x}) d \mathbf{x}=\int \ldots \int \frac{w\left(x_{1}, \mathbf{y}\right)}{\partial G / \partial x_{1}} d \mathbf{y}+O_{S}(\varepsilon),
$$

as required. Moreover we remark that the positivity property claimed in the lemma is an immediate corollary of the other results.

The transition from weights in $\mathcal{C}(S)$ to those in the more restrictive class $\mathcal{C}_{0}(S)$ is accomplished using the following result. 
Lemma 12 Let $w \in \mathcal{C}(S)$, and suppose that $|\nabla G(\mathbf{x})| \geq \lambda>0$ on $\operatorname{supp}(w)$. For each $\mathbf{y}$ let

$$
w_{\delta}\left(\frac{\mathbf{x}-\mathbf{y}}{\delta}, \mathbf{y}\right)=w_{1}(\mathbf{x}),
$$

say, be the function described in Lemma 2. Then there exists a $\delta$ in the range $1 \ll_{S} \delta \leq 1$ such that for each $\mathbf{y}$ either

(i) $|G(\mathbf{x})| \leq 1 / 2$ on $\operatorname{supp}\left(w_{1}\right)$ and, after a suitable permutation of the variables $x_{1}, \ldots, x_{n}$ the function $w_{1}(\mathbf{x})$ lies in $\mathcal{C}_{0}(S, \lambda)$

or

(ii) $|G(\mathbf{x})| \gg_{S} 1$ on $\operatorname{supp}\left(w_{1}\right)$.

In applications of the lemma the second case above will correspond to trivial situations in which the weight vanishes at all points of interest.

We proceed to prove the lemma using the subdivision procedure of Lemma 2. When we arrange that $w_{1}(\mathbf{x}) \in \mathcal{C}_{0}(S, \lambda)$ we shall choose the corresponding value of $R$, as required for the definition of $\mathcal{C}_{0}$, to satisfy $\delta \leq R \leq 1$. If $w_{1}$ does not vanish identically, there is an $\mathbf{x} \in \operatorname{supp}\left(w_{1}\right) \subseteq \operatorname{supp}(w)$ for which $\|\mathbf{x}-\mathbf{y}\| \leq \delta$ and $|\nabla G(\mathbf{x})| \geq \lambda$. It follows that $|\nabla G(\mathbf{y})| \geq \lambda / 2$, providing that $\delta \ll_{S} \lambda$. There will then be at least one index $j$ such that $\left|\partial G / \partial y_{j}\right| \geq \lambda / 2 n$. The relevent values of $\mathbf{y}$ will be divided into $n$ subsets, depending on the index $j$ which occurs, and for each $j$ the values of $\mathbf{y}$ fall into two types according to the sign of $\partial G(\mathbf{y}) / \partial y_{j}$. Without loss of generality we may therefore take $j=1$ and $\partial G(\mathbf{y}) / \partial y_{1} \geq \lambda / 2 n$. Now, if $\mathbf{x} \in \operatorname{supp}\left(w_{1}\right)$ then $\left|x_{j}-y_{j}\right| \leq \delta \leq R$ for each $j$. Thus, if $\left|x_{1}^{\prime}-x_{1}\right| \leq R$, the vector

$$
\mathbf{x}^{\prime}=\left(x_{1}^{\prime}, x_{2}, \ldots, x_{n}\right)=\left(x_{1}^{\prime}, x_{2}^{\prime}, \ldots, x_{n}^{\prime}\right)
$$

satisfies $\left|x_{j}^{\prime}-y_{j}\right| \leq 2 R$ for each $j$. It then follows that

$$
\left.\frac{\partial G}{\partial x_{1}}\right|_{\mathbf{x}=\mathbf{x}^{\prime}}=\left.\frac{\partial G}{\partial x_{1}}\right|_{\mathbf{x}=\mathbf{y}}+O_{S}(R),
$$

whence

$$
\left.\frac{\partial G}{\partial x_{1}}\right|_{\mathbf{x}=\mathbf{x}^{\prime}} \geq \frac{\lambda}{4 n}
$$

if $R \ll_{S} \lambda$. Thus (6.1) is satisfied for such $R$.

To handle the second condition we introduce a positive parameter $\eta$, satisfying $1 \ll_{S} \eta \leq 1$, which will be specified in due course. We proceed to investigate two cases, depending on whether $|G(\mathbf{y})| \geq \eta$ or not. We begin by observing that $|G(\mathbf{y})-G(\mathbf{x})| \ll_{S} \delta$, if $\left|x_{j}-y_{j}\right| \leq \delta$ for each $j$. Hence if $\delta \ll_{S} \eta$ then $|G(\mathbf{y})-G(\mathbf{x})| \leq \eta / 2$ for all $\mathbf{x} \in \operatorname{supp}\left(w_{1}\right)$. It follows that if $|G(\mathbf{y})| \geq \eta$ then $|G(\mathbf{x})| \geq \eta / 2$ throughout $\operatorname{supp}\left(w_{1}\right)$. This gives us the second case of the lemma, providing that $\eta \gg_{S} 1$. On the other hand, if $|G(\mathbf{y})|<\eta$, then $|G(\mathbf{z})| \leq 2 \eta$ for $\mathbf{z} \in \operatorname{supp}\left(w_{1}\right)$. Hence if $x_{j}=z_{j}$ for $j \geq 2$, the function $G(\mathbf{x})$ must vanish for some $x_{1}$ in the range $\left|x_{1}-z_{1}\right| \leq R$, in view of the lower bound (6.4), providing 
that $2 \eta \leq R \frac{\lambda}{4 n}$. This leads to the first case of the lemma. It remains to specify appropriate choices for $\delta, R$ and $\eta$. We write the conditions required as

$$
\begin{gathered}
\delta \leq R \leq 1, \quad \delta \leq C_{1}(S) \lambda, \quad R \leq C_{2}(S) \lambda, \\
1 \ll S \eta \leq 1, \quad \delta \leq C_{3}(S) \eta, \text { and } \eta \leq \frac{R \lambda}{8 n},
\end{gathered}
$$

and we set

$$
R=\min \left\{1, C_{2}(S) \lambda\right\}, \quad \eta=\min \left\{1, R \frac{\lambda}{8 n}\right\}
$$

and

$$
\delta=\min \left\{R, C_{1}(S) \lambda, C_{3}(S) \eta\right\} .
$$

These choices do indeed yield $\delta \gg_{S} 1$ as required. This completes the proof of Lemma 12.

We can now observe that it suffices to prove Theorems $4-8$ for weights $w_{1} \in$ $\mathcal{C}_{0}(S)$. In the first place we note that if

$$
w(\mathbf{x})=\delta^{-n} \int w_{\delta}\left(\frac{\mathbf{x}-\mathbf{y}}{\delta}, \mathbf{y}\right) d \mathbf{y}
$$

then $w_{1}(\mathbf{x})$ is in $\mathcal{C}(S)$, and $w_{1}(\mathbf{x})$ vanishes unless $|\mathbf{y}| \leq R \ll_{S} 1$, for a suitable $R$. Moreover

$$
N(F, w(\mathbf{x}))=\delta^{-n} \int N\left(F, w_{\delta}\left(\frac{\mathbf{x}-\mathbf{y}}{\delta}, \mathbf{y}\right)\right) d \mathbf{y},
$$

and

$$
\sigma_{\infty}(G, w(\mathbf{x}))=\delta^{-n} \int \sigma_{\infty}\left(G, w_{\delta}\left(\frac{\mathbf{x}-\mathbf{y}}{\delta}, \mathbf{y}\right)\right) d \mathbf{y}
$$

since the convergence of

$$
\frac{1}{2 \varepsilon} \int_{|G(\mathbf{x})| \leq \varepsilon} w_{1}(\mathbf{x}) d \mathbf{x}
$$

is uniform with respect to $\mathbf{y}$, whether case (i) or case (ii) of Lemma 12 applies. Finally, the error terms in each of the theorems behave correctly, since they take the form $O_{S, \varepsilon}\left(P^{K+\varepsilon}\right)$ for the weights $w_{1}(\mathbf{x})$, and

$$
\delta^{-n} \int_{|\mathbf{y}| \leq R} O_{S, \varepsilon}\left(P^{K+\varepsilon}\right) d \mathbf{y} \ll_{S, \varepsilon} P^{K+\varepsilon} .
$$

\section{The Integral $I_{q}(\mathbf{c})$ - Simple Estimates}

In the next two sections we shall consider the integral

$$
\begin{aligned}
I_{q}(\mathbf{c}) & =\int_{\mathbb{R}^{n}} w\left(P^{-1} \mathbf{x}\right) h\left(Q^{-1} q, Q^{-2} F(\mathbf{x})\right) e_{q}(-\mathbf{c} \cdot \mathbf{x}) d \mathbf{x} \\
& =P^{n} \int_{\mathbb{R}^{n}} w(\mathbf{x}) h\left(Q^{-1} q, Q^{-2} P^{d} G(\mathbf{x})\right) e_{q}(-P \mathbf{c} . \mathbf{x}) d \mathbf{x} .
\end{aligned}
$$


The behaviour of the function $h$, as described in Lemmas 4 and 5 , makes it natural to choose $Q=P^{d / 2}$, which we now do. The function $h$ in the above integral now becomes $h\left(Q^{-1} q, G(\mathbf{x})\right)$. If $\mathbf{x} \in \operatorname{supp}(w)$ this will vanish for $q \ll_{S} Q$, by Lemma 4 , since

$$
G(\mathbf{x}) \ll_{S} 1
$$

for such $\mathbf{x}$. It is now natural to introduce the definition

$$
I_{r}^{*}(\mathbf{v})=\int_{\mathbb{R}^{n}} w(\mathbf{x}) h(r, G(\mathbf{x})) e_{r}(-\mathbf{v} \cdot \mathbf{x}) d \mathbf{x},
$$

so that

$$
I_{q}(\mathbf{c})=P^{n} I_{r}^{*}(\mathbf{v}), \quad\left(r=Q^{-1} q, \mathbf{v}=Q^{-1} P \mathbf{c}\right) .
$$

The goal of the present section and the next is to examine $I_{r}^{*}(\mathbf{v})$ in detail. In the case in which $F$ is homogeneous we will want to perform partial summation with respect to $q$. We will therefore need to know how the derivative $\partial I_{r}^{*}(\mathbf{v}) / \partial r$ behaves in this case.

We begin by examining the case $\mathbf{c}=\mathbf{0}$, which relates to the singular integral, as we shall see in due course. We may, of course, assume that $q \ll Q$, or equivalently that $r \ll 1$. Since we are supposing that $w \in \mathcal{C}_{0}(S)$ we have

$$
\frac{\partial G}{\partial x_{1}} \gg_{S} 1
$$

on $\operatorname{supp}(w)$. We may therefore substitute $y=G(\mathbf{x})$ for $x_{1}$ in the integral

$$
\int w(\mathbf{x}) h(r, G(\mathbf{x})) d \mathbf{x}
$$

to give

$$
\int I(y) h(r, y) d y
$$

where

$$
I(y)=\int w(\mathbf{x}) \frac{d \mathbf{z}}{\partial G / \partial x_{1}} .
$$

In this last integral we have written $\mathbf{z}=\left(x_{2}, \ldots, x_{n}\right)$, and we have defined $x_{1}$ by the relation $G(\mathbf{x})=y$. We now observe that

$$
\frac{\partial x_{1}}{\partial y}=\left(\frac{\partial G}{\partial x_{1}}\right)^{-1}
$$

This allows us to compute

$$
\frac{\partial^{n}}{\partial y^{n}} \frac{w\left(x_{1}(y), \mathbf{z}\right)}{\partial G\left(x_{1}(y), \mathbf{z}\right) / \partial x_{1}} .
$$

Since $\partial G / \partial x_{1} \gg_{S} 1$ on $\operatorname{supp}(w)$, and all higher derivatives are $O_{S}(1)$, we deduce that $I(y) \in \mathcal{C}(S)$. It therefore follows from Lemma 9 that

$$
\int I(y) h(r, y) d y=I(0)+O_{S, N}\left(r^{N}\right)
$$


for any $N>0$. Since Theorem 3 yields $I(0)=\sigma_{\infty}(G, w)$ we arrive at the following result.

Lemma 13 Suppose that $w \in \mathcal{C}_{0}(S)$. Then

$$
I_{r}^{*}(\mathbf{0})=\sigma_{\infty}(G, w)+O_{S, N}\left(r^{N}\right)
$$

for any $N>0$, providing that $r \ll 1$. Consequently

$$
I_{q}(\mathbf{0})=P^{n}\left\{\sigma_{\infty}(G, w)+O_{S, N}\left((q / Q)^{N}\right)\right\}
$$

for $q \ll Q$.

We turn now to upper bounds for $I_{r}^{*}(\mathbf{v})$. In this section we shall give only some rather simple estimates. In the next section we shall investigate more sophisticated bounds.

To deal succinctly with the various partial derivatives of $h$ which we shall encounter, we introduce the class of functions $\mathcal{H}$. This will be the set of infinitely differentiable functions $f:(0, \infty) \times \mathbb{R} \rightarrow \mathbb{C}$ such that for any positive integer $N$ there are positive real numbers $K_{0, N}, K_{1, N}, \ldots$ for which $f$ satisfies the inequalities

$$
\begin{gathered}
|f(r, x)| \leq K_{0, N}\left(r^{N}+\min \left\{1,\left(\frac{r}{|x|}\right)^{N}\right\}\right), \\
\left|\frac{\partial^{j} f(r, x)}{\partial x^{j}}\right| \leq K_{j, N} r^{-j} \min \left\{1,\left(\frac{r}{|x|}\right)^{N}\right\}, \quad j \geq 1 .
\end{gathered}
$$

Only a finite number of different functions $f \in \mathcal{H}$ will arise in our analysis, and for each function there will be a corresponding set of absolute constants $K_{j, N}$. Where no ambiguity arises we shall abbreviate $f(r, x)$ to $f(x)$.

Our first goal is to show, in the homogeneous case, that $\partial I_{q}(\mathbf{c}) / \partial q$ may be estimated via an integral similar to $I_{q}(\mathbf{c})$ itself. We state the result in terms of $I_{r}^{*}(\mathbf{v})$

\section{Lemma 14 We have}

$$
I_{r}^{*}(\mathbf{v}) \ll r^{-1}\left|I\left(r^{-1} \mathbf{v}\right)\right|,
$$

where

$$
I(\mathbf{u})=I(r ; \mathbf{u})=\int_{\mathbb{R}^{n}} w_{1}(\mathbf{x}) f(G(\mathbf{x})) e(-\mathbf{u} \cdot \mathbf{x}) d \mathbf{x},
$$

for appropriate functions $f(*)=f(r, *) \in \mathcal{H}$ and $w_{1} \in \mathcal{C}_{0}(S)$, with $\operatorname{supp}\left(w_{1}\right) \subseteq$ $\operatorname{supp}(w)$. Moreover, in the homogeneous case, or if $\mathbf{v}=\mathbf{0}$, we have

$$
\frac{\partial I_{r}^{*}(\mathbf{v})}{\partial r} \ll r^{-2}\left|I\left(r^{-1} \mathbf{v}\right)\right|,
$$

again for appropriate functions $f \in \mathcal{H}$ and $w_{1} \in \mathcal{C}_{0}(S)$. 
In view of Lemma 5 we see that

$$
f(r, x)=r^{k+1} \frac{\partial^{k} h(r, x)}{\partial r^{k}}
$$

is in $\mathcal{H}$, for $k=0$ or 1 . The first statement of the lemma is therefore immediate, so we turn our attention to the derivative of $I_{r}^{*}(\mathbf{v})$. We begin by observing that

$$
\frac{\partial h(r, F) e_{r}(-\mathbf{v} \cdot \mathbf{x})}{\partial r}=\frac{\partial h(r, F)}{\partial r} e_{r}(-\mathbf{v} \cdot \mathbf{x})-r^{-1} h(r, F) e_{r}(-\mathbf{v} \cdot \mathbf{x})\left(\frac{-2 \pi i \mathbf{v} \cdot \mathbf{x}}{r}\right) .
$$

It then follows that

$$
\frac{\partial I_{r}^{*}(\mathbf{v})}{\partial r} \ll r^{-2}\left|I^{(k)}\left(r^{-1} \mathbf{v}\right)\right|,
$$

for either $k=0$ or $k=1$, where

$$
I^{(k)}(\mathbf{u})=\int_{\mathbb{R}^{n}} w(\mathbf{x}) f(G(\mathbf{x})) e(-\mathbf{u} \cdot \mathbf{x})(-2 \pi i \mathbf{u} \cdot \mathbf{x})^{k} d \mathbf{x},
$$

for an appropriate function $f \in \mathcal{H}$.

If $k=0$, or if $\mathbf{v}=\mathbf{0}$, the lemma follows at once, so we examine the case in which $k=1$ and $G$ is homogeneous. Our main task is to remove the factor $-2 \pi i \mathbf{u} . \mathbf{x}$. In general, if $g(\mathbf{x})$ is a smooth function of compact support, the divergence theorem yields

$$
\int_{\mathbb{R}^{n}} \nabla \cdot\{g(\mathbf{x}) e(-\mathbf{u} \cdot \mathbf{x}) \mathbf{x}\} d \mathbf{x}=0 .
$$

However the integral on the left is

$$
\int_{\mathbb{R}^{n}} g(\mathbf{x}) e(-\mathbf{u} \cdot \mathbf{x})(-2 \pi i \mathbf{u} \cdot \mathbf{x}) d \mathbf{x}+\int_{\mathbb{R}^{n}}\{n g(\mathbf{x})+(\mathbf{x} \cdot \nabla) g(\mathbf{x})\} e(-\mathbf{u} \cdot \mathbf{x}) d \mathbf{x} .
$$

If

$$
g(\mathbf{x})=w(\mathbf{x}) f(G(\mathbf{x}))
$$

then

$$
(\mathbf{x} . \nabla) g(\mathbf{x})=\{(\mathbf{x} \cdot \nabla) w(\mathbf{x})\} f(G(\mathbf{x}))+w(\mathbf{x})\{(\mathbf{x} \cdot \nabla) G(\mathbf{x})\} f^{\prime}(G(\mathbf{x})) .
$$

Here

$$
(\mathbf{x} . \nabla) G(\mathbf{x})=d G(\mathbf{x}),
$$

since $G$ is homogeneous. Moreover $(\mathbf{x} . \nabla) w(\mathbf{x}) \in \mathcal{C}_{0}(S)$, and $x f^{\prime}(x)$ is in $\mathcal{H}$ whenever $f$ is. The lemma therefore follows on comparing estimates.

We now have to estimate $I(\mathbf{u})=I(r ; \mathbf{u})$. We first record the trivial bound, which follows from the estimate (7.1).

Lemma 15 We have

$$
I(r ; \mathbf{u}) \ll_{S} r
$$

for any vector $\mathbf{u}$. 
To prove this we observe that

$$
I(\mathbf{u}) \ll \int\left\{r+\min \left(1, \frac{r^{2}}{G(\mathbf{x})^{2}}\right)\right\} d \mathbf{x},
$$

the integral being over the support of $w(\mathbf{x})$. Since

$$
\frac{\partial G}{\partial x_{1}} \gg_{S} 1
$$

in the latter region, one sees that the measure of the set where $|G(\mathbf{x})| \leq \lambda$ is $O_{S}(\lambda)$. The integral above is therefore $O_{S}(r)$, which suffices for the lemma.

As a corollary of Lemmas 14 and 15 we have the following estimate for the case $\mathbf{c}=\mathbf{0}$.

Lemma 16 Suppose that $w \in \mathcal{C}_{0}(S)$. Then

$$
\frac{\partial^{j} I_{q}(\mathbf{0})}{\partial q^{j}} \ll_{S} P^{n} q^{-j}, \quad(j=0,1) .
$$

We now proceed to a more detailed analysis of $I(\mathbf{u})$. Since the function $f$ need not have compact support we begin by choosing $K$ with $1 \ll_{S} K \ll_{S} 1$, such that $|G(\mathbf{x})| \leq K$ on $\operatorname{supp}\left(w_{1}\right)$. We then set $w_{2}(t)=w_{0}(t / 2 K)$, where $w_{0}$ is given by $(2.1)$. Since $w_{2}(G(\mathbf{x})) \gg 1$ on $\operatorname{supp}\left(w_{1}\right)$, an application of Lemma 3 then shows that

$$
w_{3}(\mathbf{x})=\frac{w_{1}(\mathbf{x})}{w_{2}(G(\mathbf{x}))}
$$

belongs to $\mathcal{C}_{0}(S)$. We now express $I(\mathbf{u})$ in the shape

$$
\int_{\mathbb{R}^{n}} w_{3}(\mathbf{x})\left\{w_{2}(G) f(G)\right\} e(-\mathbf{u} \cdot \mathbf{x}) d \mathbf{x},
$$

and we use the Fourier transform to write

$$
w_{2}(G) f(G)=\int_{-\infty}^{\infty} p(t) e(t G) d t,
$$

where

$$
p(t)=\int_{-\infty}^{\infty} w_{2}(v) f(v) e(-t v) d v .
$$

This yields

$$
I(\mathbf{u})=\int_{-\infty}^{\infty} p(t) \int_{\mathbb{R}^{n}} w_{3}(\mathbf{x}) e(t G(\mathbf{x})-\mathbf{u} \cdot \mathbf{x}) d \mathbf{x} d t .
$$

Since

we see that

$$
\frac{d^{N}}{d v^{N}} w_{2}(v) f(v) \ll_{N} r^{-N} \min \left\{1,\left(\frac{r}{|v|}\right)^{2}\right\},
$$

$$
p(t) \ll_{N} r(r|t|)^{-N}
$$

for any $N \geq 0$, on repeated integration by parts. We summarize our findings as follows. 
Lemma 17 There exist weights $w_{2}, w_{3} \in \mathcal{C}_{0}(S)$, with $\operatorname{supp}\left(w_{3}\right) \subseteq \operatorname{supp}(w)$, such that if $p$ is the Fourier transform of $w_{2}(x) f(x)$ then

$$
I(r ; \mathbf{u})=\int_{-\infty}^{\infty} p(t) \int_{\mathbb{R}^{n}} w_{3}(\mathbf{x}) e(t G(\mathbf{x})-\mathbf{u} \cdot \mathbf{x}) d \mathbf{x} d t
$$

and

$$
p(t) \ll_{N} r(r|t|)^{-N}
$$

for any $N \geq 0$.

We end this section by using Lemma 17 to establish a straightforward bound for $I(\mathbf{u})$. When $\mathbf{u}$ is large we do apply Lemma 10, which shows that

$$
\int_{\mathbb{R}^{n}} w_{3}(\mathbf{x}) e(t G(\mathbf{x})-\mathbf{u} \cdot \mathbf{x}) d \mathbf{x} \ll_{S}|\mathbf{u}|^{-M},
$$

for any $M>0$, whenever $|\mathbf{u}| \gg_{S}|t|$. In the alternative case, when $\mathbf{u}$ is small, we shall use the trivial estimate $O_{S}(1)$. The bound (7.3), with $N=0$ for $|t| \ll_{S}|\mathbf{u}|$ and $N=M$ otherwise, then shows that

$$
I(\mathbf{u}) \ll_{S}|\mathbf{u}|^{1-M} r+|\mathbf{u}|^{1-M} r^{1-M} \ll_{S}|\mathbf{u}|^{1-M} r^{1-M},
$$

bearing in mind that $r \ll_{S} 1$. We therefore have the following estimate.

Lemma 18 For any $N \geq 0$ we have

$$
I(r ; \mathbf{u}) \ll_{N, S} r^{-N}|\mathbf{u}|^{-N} .
$$

Lemmas 14 and 18 have the following immediate corollary when $d=2$, in which case $Q=P$.

Lemma 19 When $d=2$ and $\mathbf{c} \neq \mathbf{0}$ we have

$$
I_{q}(\mathbf{c}) \ll_{S, N} P^{n+1} q^{-1}|\mathbf{c}|^{-N},
$$

for any $N>0$.

This shows that $\mathbf{c}$ with $|\mathbf{c}| \gg P^{\varepsilon}$ will make a negligible contribution in our analysis.

\section{The Integral $I_{q}(\mathbf{c})$-Harder Estimates}

Before proceeding further it may be of interest to reflect on the way the estimate (7.2) relates to that which arises in the classical circle method. The term $c_{Q} Q^{-2} q^{-n} S_{q}(\mathbf{c}) I_{q}^{(0)}(\mathbf{c})$ in Theorem 2 corresponds to the total contribution from the Farey arcs of denominator $q$. In particular, a little thought shows that $Q^{-2} I_{q}(\mathbf{c})$ should be compared with

$$
\int_{-1 / q Q}^{1 / q Q}\left\{\int_{\mathbb{R}^{n}} w\left(P^{-1} \mathbf{x}\right) e\left(t F(\mathbf{x})-\frac{\mathbf{c} \cdot \mathbf{x}}{q}\right) d \mathbf{x}\right\} d t,
$$


and hence that $I(\mathbf{u})$ should be compared with

$$
r \int_{-1 / r}^{1 / r}\left\{\int_{\mathbb{R}^{n}} w(\mathbf{x}) e(t G(\mathbf{x})-\mathbf{u} \cdot \mathbf{x}) d \mathbf{x}\right\} d t
$$

In the first of the above integrals with respect to $t$ the range corresponds to an integration over a Farey arc $[a / q-1 / q Q, a / q+1 / q Q]$, and in the second integral this has been re-scaled by a factor $Q^{2}$. Of course the condition (7.3) shows that the integral (7.2) is effectively for $t \ll r^{-1}=Q / q$ too.

We now turn to our next estimate for $I(\mathbf{u})$. We shall express our results in terms of a parameter $R \geq 1$, which we shall take to be sufficiently large in terms of the set $S$. In view of Lemma 18 we shall assume here that $|\mathbf{u}| \leq r^{-1} R$, for otherwise $I(\mathbf{u})$ will be negligible. We cannot expect any non-trivial bound for $I(\mathbf{u})$ unless $|\mathbf{u}| \geq 1$, which we may therefore assume with no loss of generality. It turns out that it is convenient to impose the slightly stronger condition

$$
|\mathbf{u}| \geq R^{3}
$$

Notice that our two assumptions together require that $r \leq R^{-2}$. We begin by using the subdivision process of Lemma 2 to split up the range for $\mathbf{x}$. This will require a parameter $\delta>0$, whose definition we shall give in due course. We then find that

$$
I(\mathbf{u})=\delta^{-n} \int_{-\infty}^{\infty} p(t) \int_{\mathbb{R}^{n}} \int_{\mathbb{R}^{n}} w_{\delta}\left(\frac{\mathbf{x}-\mathbf{y}}{\delta}, \mathbf{y}\right) e(t G(\mathbf{x})-\mathbf{u} \cdot \mathbf{x}) d \mathbf{x} d \mathbf{y} d t,
$$

where

$$
w_{3}(\mathbf{x})=\delta^{-n} \int w_{\delta}\left(\frac{\mathbf{x}-\mathbf{y}}{\delta}, \mathbf{y}\right) d \mathbf{y} .
$$

Hence, if we substitute $\mathbf{x}=\mathbf{y}+\delta \mathbf{z}$ we find that

$$
I(\mathbf{u}) \leq \int_{\mathbb{R}^{n}} \int_{-\infty}^{\infty}|p(t)|\left|\int_{\mathbb{R}^{n}} w_{4}(\mathbf{z}) e(t G(\mathbf{x})-\mathbf{u} . \mathbf{x}) d \mathbf{z}\right| d t d \mathbf{y}
$$

with

$$
w_{4}(\mathbf{z})=w_{4}(\mathbf{z}, \mathbf{y})=w_{\delta}(\mathbf{z}, \mathbf{y}) \in \mathcal{C}_{0}(S)
$$

Here we have $\mathbf{x} \in \operatorname{supp}\left(w_{3}\right) \subseteq \operatorname{supp}(w)$ whenever $w_{4} \neq 0$. Moreover $\mathbf{y}$ runs over a range $\mathbf{y} \ll_{S} 1$. We shall perform the two outer integration trivially. We therefore consider $\mathbf{y}$ and $t$ to be fixed in the following. For most values of $(\mathbf{y}, t)$ it will turn out that the innermost integral above is negligible. We shall call such pairs $(\mathbf{y}, t)$ 'good'. For the remaining 'bad' values of $(\mathbf{y}, t)$ we shall estimate the innermost integral trivially.

With the above discussion in mind we apply Lemma 10 to the innermost integral in (8.2), taking

$$
f(\mathbf{z})=t G(\mathbf{y}+\delta \mathbf{z})-\mathbf{u} \cdot(\mathbf{y}+\delta \mathbf{z}) .
$$


The partial derivatives of order $k \geq 2$ are $O_{S, k}\left(|t| \delta^{k}\right)$. Moreover

$$
|\nabla f(\mathbf{z})|=|\nabla f(\mathbf{0})|+O_{S}\left(|t| \delta^{2}\right) \gg|\nabla f(\mathbf{0})|
$$

providing that

$$
|\nabla f(\mathbf{0})| \geq|t| \delta^{2} R
$$

It follows that

$$
\int_{\mathbb{R}^{n}} w_{4}(\mathbf{z}) e(t G(\mathbf{x})-\mathbf{u} \cdot \mathbf{x}) d \mathbf{z} \ll_{S, N} R^{-N}
$$

whenever

$$
|\nabla f(\mathbf{0})| \geq R \max \left\{|t| \delta^{2}, 1\right\} .
$$

The natural choice of $\delta$ is therefore $\delta=\min \left\{1,|t|^{-1 / 2}\right\}$. However it turns out that it is more convenient to set $\delta=|\mathbf{u}|^{-1 / 2}$ which will be essentially equivalent to the optimal value above. We can now define $(\mathbf{y}, t)$ to be 'good' if

$$
|\nabla f(\mathbf{0})|=|\mathbf{u}|^{-1 / 2}|t \nabla G(\mathbf{y})-\mathbf{u}| \geq R \max \{|t| /|\mathbf{u}|, 1\} .
$$

In view of the estimate (7.3) it follows that the total contribution to (8.2) from such $(\mathbf{y}, t)$ is $O_{S}\left(R^{-N}\right)$.

In the alternative case, when $(\mathbf{y}, t)$ is 'bad', we have

$$
|t \nabla G(\mathbf{y})-\mathbf{u}| \leq R|\mathbf{u}|^{1 / 2} \max \{|t| /|\mathbf{u}|, 1\} .
$$

We see from the construction of $w_{\delta}$, in $\S 2$, that any relevent $\mathbf{y}$ is within $\delta$ of some point of $\operatorname{supp}(w)$. Since $\delta=|\mathbf{u}|^{-1 / 2} \leq R^{-3 / 2}$ it follows that $|\nabla G(\mathbf{y})| \gg_{S} 1$ if $R$ is sufficiently large in terms of $S$. As $\nabla G(\mathbf{y})$ is trivially $O_{S}(1)$ and $|\mathbf{u}| \geq R^{3}$, we see that we must have $|\mathbf{u}| \ll_{S}|t| \ll_{S}|\mathbf{u}|$, so that

$$
|t \nabla G(\mathbf{y})-\mathbf{u}| \ll_{S} R|\mathbf{u}|^{1 / 2}
$$

for all 'bad' $(\mathbf{y}, t)$. We now see that

$$
I(\mathbf{u}) \ll_{S, N} R^{-N}+\int_{\mathbf{y}} \int_{-\infty}^{\infty}|p(t)| \int_{\mathbf{z}}\left|w_{4}(\mathbf{z})\right| d \mathbf{z} d t d \mathbf{y},
$$

where $(\mathbf{y}, t)$ runs over 'bad' values, with $\mathbf{y} \ll_{S} 1$. We now substitute $\mathbf{x}=\mathbf{y}+\delta \mathbf{z}$ for $\mathbf{y}$, and observe that

$$
t \nabla G(\mathbf{x})-t \nabla G(\mathbf{y}) \ll_{S}|t| \delta \ll_{S}|\mathbf{u}|^{1 / 2} \ll_{S} R|\mathbf{u}|^{1 / 2},
$$

since $\mathbf{z} \ll_{S} 1$ and $t \ll_{S}|\mathbf{u}|$. Thus if $\mathbf{y}$ satisfies (8.3), then so must $\mathbf{x}$, with a different implied constant. Moreover $w_{4}(\mathbf{z}) \neq 0$ implies that $\mathbf{x} \in \operatorname{supp}(w)$, and that $\mathbf{z} \ll_{S}$ 1. Since (7.3) yields $p(t) \ll_{S} r$, we may now conclude as follows.

Lemma 20 If $|\mathbf{u}| \geq R^{3}$ then

$$
I(\mathbf{u}) \ll_{S, N} R^{-N}+r|\mathbf{u}| \operatorname{meas}(\mathcal{S}) .
$$


Here

$$
\mathcal{S}=\left\{\mathbf{x} \in \operatorname{supp}(w):|t \nabla G(\mathbf{x})-\mathbf{u}| \leq C R|\mathbf{u}|^{1 / 2}\right\}
$$

for an appropriate value of $t$ in the range

$$
A|\mathbf{u}| \leq|t| \leq B|\mathbf{u}|
$$

with appropriate positive constants $A=A(S), B=B(S)$ and $C=C(S)$.

Notice that we have dropped the condition $r|\mathbf{u}| \leq R$, since the bound of the lemma follows immediately from Lemma 18 when $r|\mathbf{u}| \geq R$.

We must now discuss the set $\mathcal{S}$. It turns out that the size of meas $(\mathcal{S})$ depends heavily on the behaviour of the Hessian matrix

$$
\mathbf{H}(\mathbf{x})_{i j}=\frac{\partial^{2}}{\partial x_{i} \partial x_{j}} G(\mathbf{x})
$$

and its determinant, $\operatorname{det} \mathbf{H}(\mathbf{x})$. We shall write $H(\mathbf{x})$ for $|\operatorname{det} \mathbf{H}(\mathbf{x})|$. When $F^{(0)}$ is a quadratic form this merely produces $2^{n}\left|\operatorname{det} F^{(0)}\right|$, and the situation is quite simple, but for forms of higher degree things are far more complex. When the Hessian is well behaved we can estimate meas $(\mathcal{S})$ in the following simple way.

Lemma 21 Suppose that $H(\mathbf{x}) \gg_{S} 1$ for all $\mathbf{x} \in \operatorname{supp}(w)$. Then

$$
\operatorname{meas}(\mathcal{S}) \ll_{S}|\mathbf{u}|^{-n / 2} R^{n} \text {. }
$$

As a corollary we shall show that Lemmas 20 and 21 yield:-

Lemma 22 Suppose that $n \geq 3$, and that the Hessian condition of Lemma 21 holds. Then for any $\varepsilon \in(0,1 / 2)$ we have

$$
I(r ; \mathbf{u}) \ll_{S, \varepsilon}\left(r^{-1}|\mathbf{u}|\right)^{\varepsilon} r|\mathbf{u}|^{1-n / 2},
$$

and hence

$$
I_{q}(\mathbf{c}) \ll_{S, \varepsilon} P^{n}\left(\frac{P Q|\mathbf{c}|}{q^{2}}\right)^{\varepsilon}\left(\frac{P|\mathbf{c}|}{q}\right)^{1-n / 2} .
$$

Moreover in the homogeneous case the same bound applies to $q(\partial / \partial q) I_{q}(\mathbf{c})$. These estimates hold, in particular, if $n \geq 3$ and $F^{(0)}$ is a non-singular quadratic form.

Note here that there is no size constraint on $\mathbf{u}$.

To establish Lemma 21 we observe that if $\mathbf{x}$ and $\mathbf{x}+\mathbf{x}^{\prime}$ are in $\mathcal{S}$, then

$$
\nabla G\left(\mathbf{x}+\mathbf{x}^{\prime}\right)-\nabla G(\mathbf{x}) \ll R|\mathbf{u}|^{-1 / 2} .
$$

However

$$
\nabla G\left(\mathbf{x}+\mathbf{x}^{\prime}\right)-\nabla G(\mathbf{x})=\mathbf{H}(\mathbf{x}) \mathbf{x}^{\prime}+O_{S}\left(\left|\mathbf{x}^{\prime}\right|^{2}\right) .
$$

Using the inverse of $\mathbf{H}(\mathbf{x})$, whose entries are $O_{S}(1)$ by our hypothesis, we find that

$$
\mathbf{x}^{\prime} \ll_{S} R|\mathbf{u}|^{-1 / 2}+\left|\mathbf{x}^{\prime}\right|^{2} .
$$


It follows that either $\left|\mathbf{x}^{\prime}\right|>K(S)$, for some constant $K(S)$, or $\left|\mathbf{x}^{\prime}\right| \ll_{S} R|\mathbf{u}|^{-1 / 2}$. We therefore subdivide $\operatorname{supp}(w)$ using hypercubes of side $K(S)$ to produce $O_{S}(1)$ subsets in each of which the points of $\mathcal{S}$ are restricted to a hypersphere of radius $O_{S}\left(R|\mathbf{u}|^{-1 / 2}\right)$. Lemma 21 now follows.

To prove Lemma 22 we observe that if $|\mathbf{u}| \ll_{S} r^{-2 \varepsilon / n}$ then

$$
|\mathbf{u}|^{n / 2-1-\varepsilon} \ll S r^{-\varepsilon},
$$

since

$$
0<\frac{n}{2}-1-\varepsilon<\frac{n}{2}
$$

We may therefore deduce that

$$
r \ll\left(r^{-1}|\mathbf{u}|\right)^{\varepsilon} r|\mathbf{u}|^{1-n / 2}
$$

in this case, so that the required estimate follows from Lemma 15. When $|\mathbf{u}| \gg r^{-2 \varepsilon / n}$ we apply Lemma 20 with $R=A_{S}\left(r^{-1}|\mathbf{u}|\right)^{\varepsilon / 3 n}$, for a suitably large constant $A_{S}$. Here the condition $|\mathbf{u}| \geq R^{3}$ is equivalent to $|\mathbf{u}| \gg_{S} r^{-\varepsilon /(n-\varepsilon)}$, which is satisfactory, since $2 \varepsilon / n \geq \varepsilon /(n-\varepsilon)$. Thus Lemma 21 yields

$$
I(\mathbf{u}) \ll_{S, N} R^{-N}+r|\mathbf{u}|^{1-n / 2} R^{n},
$$

Here we will automatically have $R^{n} \ll_{S}\left(r^{-1}|\mathbf{u}|\right)^{\varepsilon}$, by our choice of $R$, and $R^{-N} \ll_{S}\left(r^{-1}|\mathbf{u}|\right)^{\varepsilon} r|\mathbf{u}|^{1-n / 2}$ providing that

$$
\frac{\varepsilon}{3 n} N \geq \max \left(\frac{n}{2}-1-\varepsilon, 1-\varepsilon\right),
$$

as we may suppose.

\section{The Sum $S_{q}(\mathbf{c})$ - General Considerations}

We begin this section by recording the standard multiplicativity property.

Lemma 23 If $(u, v)=1$ then

$$
S_{u v}(\mathbf{c})=S_{u}(\bar{v} \mathbf{c}) S_{v}(\bar{u} \mathbf{c}),
$$

where $\bar{u}$ and $\bar{v}$ are defined by the congruences

$$
u \bar{u} \equiv 1(\bmod v), v \bar{v} \equiv 1(\bmod u)
$$

To prove this we write

$$
S_{u v}(\mathbf{c})=\sum_{a(\bmod u v)} * \sum_{\mathbf{b}(\bmod u v)} e_{u v}(a F(\mathbf{b})+\mathbf{c} . \mathbf{b}) .
$$

We then set $a=u a_{v}+v a_{u}$, where $a_{u}$ and $a_{v}$ run modulo $u$ and $v$ respectively, and

$$
\mathbf{b}=u \bar{u} \mathbf{b}_{v}+v \bar{v} \mathbf{b}_{u}
$$

where $\mathbf{b}_{u}$ and $\mathbf{b}_{v}$ run modulo $u$ and $v$ respectively. The result then follows.

Our second result makes a first step towards handling the case in which $q$ is square-full. 
Lemma 24 Let $t \geq 2$ and $s=[t / 2]$. Then

$$
S_{p^{t}}(\mathbf{c})=p^{s(n+1)} \sum_{d\left(\bmod p^{t-s}\right)}^{*} \quad \sum_{\mathbf{x}\left(\bmod p^{t-s}\right)}^{(1)} e_{p^{t}}(d F(\mathbf{x})+\mathbf{x . c}),
$$

where $\Sigma^{(1)}$ indicates the conditions $p^{s} \mid F(\mathbf{x})$ and $p^{s} \mid d \nabla F(\mathbf{x})+\mathbf{c}$.

For the proof we substitute $a=d+p^{t-s} f$ in the definition of $S_{p^{t}}(\mathbf{c})$ so that

$$
\begin{aligned}
S_{p^{t}}(\mathbf{c}) & =\sum_{a\left(\bmod p^{t}\right)}^{*} \sum_{\mathbf{b}\left(\bmod p^{t}\right)} e_{p^{t}}(a F(\mathbf{b})+\mathbf{b . c}) \\
& =\sum_{d\left(\bmod p^{t-s}\right)}^{*} \sum_{f\left(\bmod p^{s}\right)} \sum_{\mathbf{b}\left(\bmod p^{t}\right)} e_{p^{t}}(d F(\mathbf{b})+\mathbf{b . c}) e_{p^{s}}(f F(\mathbf{b})) \\
& =p^{s} \sum_{d\left(\bmod p^{t-s}\right)}^{*} \sum_{\mathbf{b}\left(\bmod p^{t}\right), p^{s} \mid F(\mathbf{b})} e_{p^{t}}(d F(\mathbf{b})+\mathbf{b . c}) .
\end{aligned}
$$

We can now put $\mathbf{b}=\mathbf{x}+p^{t-s} \mathbf{y}$ to obtain

$$
S_{p^{t}}(\mathbf{c})=p^{s} \sum_{d\left(\bmod p^{t-s}\right)}^{*} \sum_{\mathbf{x}\left(\bmod p^{t-s}\right), p^{s} \mid F(\mathbf{x})} e_{p^{t}}(d F(\mathbf{x})+\mathbf{x . c}) \Sigma(d, \mathbf{x}),
$$

where

$$
\Sigma(d, \mathbf{x})=\sum_{\mathbf{y}\left(\bmod p^{s}\right)} e_{p^{s}}(d \mathbf{y} \cdot \nabla F(\mathbf{x})+\mathbf{y} \cdot \mathbf{c}) .
$$

Here $\Sigma(d, \mathbf{x})=0$ unless $p^{s} \mid d \nabla F(\mathbf{x})+\mathbf{c}$, in which case it is $p^{s n}$. It follows that

$$
S_{p^{t}}(\mathbf{c})=p^{s(n+1)} \sum_{d\left(\bmod p^{t-s}\right)}^{*} \quad \sum_{\mathbf{x}\left(\bmod p^{t-s}\right)}^{(1)} e_{p^{t}}(d F(\mathbf{x})+\mathbf{x . c}),
$$

as required.

\section{Quadratic Exponential Sums}

From now on we shall take $d=2$. The calculations here are all rather routine, so we shall be brief. We shall write $M$ for the matrix of the underlying quadratic form, and $\Delta=2|\operatorname{det}(M)|$. We recall that the hypotheses of our theorems require that $\Delta \neq 0$.

We begin by deducing a simple upper bound. Cauchy's inequality yields

$$
\left|S_{q}(\mathbf{c})\right|^{2} \leq \phi(q) \sum_{a(\bmod q)}^{*} \sum_{\mathbf{u}, \mathbf{v}(\bmod q)} e_{q}(a\{F(\mathbf{u})-F(\mathbf{v})\}+\{\mathbf{u}-\mathbf{v}\} . \mathbf{c}) .
$$

Substitute $\mathbf{u}=\mathbf{v}+\mathbf{w}$, so that

$$
e_{q}(a\{F(\mathbf{u})-F(\mathbf{v})\}+\{\mathbf{u}-\mathbf{v}\} . \mathbf{c})=e_{q}\left(a F^{(0)}(\mathbf{w})+\mathbf{w} . \mathbf{c}\right) e_{q}(a \mathbf{v} \cdot \nabla F(\mathbf{w})) .
$$

The summation over $\mathbf{v}$ will now produce a contribution of zero unless $q \mid \nabla F(\mathbf{w})$. However $\nabla F(\mathbf{w})=2 M \mathbf{w}$, so that this latter condition holds for $O_{\Delta}(1)$ values of $\mathbf{w}(\bmod q)$. We therefore obtain the following estimate. 
Lemma 25 We have

$$
S_{q}(\mathbf{c}) \ll_{\Delta} q^{1+n / 2} .
$$

We shall have to look in more detail at the case in which $q$ is cube-free. We begin by examining prime values of $q$. Recall that $F$ takes the form $F^{(0)}(\mathbf{x})-m$. To state our result we introduce the quadratic form $M^{-1}(\mathbf{x})$, with rational coefficients, whose matrix is $M^{-1}$. When $p \bigvee \Delta$ we may think of $M^{-1}(\mathbf{x})$ as being defined modulo $p$.

Lemma 26 Let $p \nmid \Delta$. We have $S_{p}(\mathbf{c}) \ll_{\Delta} p^{(n+1) / 2}$ except when $n$ is even and $p$ divides both $m$ and $M^{-1}(\mathbf{c})$. When $n$ is even we have

$$
S_{p}(\mathbf{c})=\left\{\begin{array}{cc}
-\left(\frac{(-1)^{n / 2} \operatorname{det}(M)}{p}\right) p^{n / 2}, & p \text { divides exactly one of } m, M^{-1}(\mathbf{c}), \\
(p-1)\left(\frac{(-1)^{n / 2} \operatorname{det}(M)}{p}\right) p^{n / 2}, & p \text { divides both of } m, M^{-1}(\mathbf{c}) .
\end{array}\right.
$$

Similarly when $n$ is odd we have

$$
S_{p}(\mathbf{c})=\left\{\begin{array}{cc}
\left(\frac{(-1)^{(n-1) / 2} \operatorname{det}(M) m}{p}\right) p^{(n+1) / 2}, & p \mid M^{-1}(\mathbf{c}), \\
\left(\frac{(-1)^{(n-1) / 2} \operatorname{det}(M) M^{-1}(\mathbf{c})}{p}\right) p^{(n+1) / 2}, & p \mid m .
\end{array}\right.
$$

In particular $S_{p}(\mathbf{c})=0$ when $n$ is odd and $p$ divides both $m$ and $M^{-1}(\mathbf{c})$.

Since $p \bigvee \Delta$, the quadratic form $F^{(0)}$ may be diagonalized, by writing

$$
R^{T} M R=\operatorname{Diag}\left(\beta_{1}, \ldots, \beta_{n}\right),
$$

say. We now substitute $\mathbf{b}=R \mathbf{x}$ and $R^{T} \mathbf{c}=\mathbf{d}$, whence

$$
\begin{aligned}
S_{p}(\mathbf{c}) & =\sum_{a=1}^{p-1} \sum_{\mathbf{b}(\bmod p)} e_{p}\left(a\left\{\mathbf{b}^{T} M \mathbf{b}-m\right\}+\mathbf{b . c}\right) \\
& =\sum_{a=1}^{p-1} \sum_{\mathbf{x}(\bmod p)} e_{p}\left(a\left\{\sum \beta_{i} x_{i}^{2}-m\right\}+\sum x_{i} d_{i}\right) \\
& =\sum_{a=1}^{p-1} e_{p}(-a m) \prod_{i=1}^{n} \sum_{x(\bmod p)} e_{p}\left(a \beta_{i} x^{2}+x d_{i}\right) .
\end{aligned}
$$

The usual formulae for Gauss sums produce

$$
\begin{aligned}
\sum_{x(\bmod p)} e_{p}\left(a \beta_{i} x^{2}+x d_{i}\right) & =\sum_{x(\bmod p)} e_{p}\left(a \beta_{i}\left\{x+d_{i} \overline{\left(2 a \beta_{i}\right)}\right\}^{2}-d_{i}^{2} \overline{\left(4 a \beta_{i}\right)}\right) \\
& =\left(\frac{a \beta_{i}}{p}\right) e_{p}\left(-d_{i}^{2} \overline{\left(4 a \beta_{i}\right)}\right) \tau_{p},
\end{aligned}
$$

where

$$
\tau_{p}=\sum_{x=1}^{p} e_{p}\left(x^{2}\right)=i^{(p-1)^{2} / 4} \sqrt{p}
$$


as usual. It therefore follows that

$$
S_{p}(\mathbf{c})=\tau_{p}^{n}\left(\frac{\operatorname{det}(M)}{p}\right) \sum_{a=1}^{p-1}\left(\frac{a}{p}\right)^{n} e_{p}\left(-a m-\sum d_{i}^{2} \overline{\left(4 a \beta_{i}\right)}\right) .
$$

However

$$
\sum \beta_{i}^{-1} d_{i}^{2}=\mathbf{d}^{T} \operatorname{Diag}\left(\beta_{1}, \ldots, \beta_{n}\right)^{-1} \mathbf{d}=\mathbf{c}^{T} R\left(R^{T} M R\right)^{-1} R^{T} \mathbf{c}=\mathbf{c}^{T} M^{-1} \mathbf{c},
$$

so that

$$
S_{p}(\mathbf{c})=\tau_{p}^{n}\left(\frac{\operatorname{det}(M)}{p}\right) K_{n}\left(-m,-\overline{4} M^{-1}(\mathbf{c}) ; p\right),
$$

where $K_{n}$ is the Kloosterman sum for $n$ even, and the Salié sum for $n$ odd. The first part of the lemma therefore follows from the standard bound

$$
\left|K_{n}(a, b ; p)\right| \leq 2 p^{1 / 2}(a, b, p)^{1 / 2} .
$$

When $n$ is even, we have $K_{n}=-1$ if $p$ divides exactly one of $a, b$, and $K_{n}=p-1$ if $p$ divides both $a$ and $b$. Similarly when $n$ is odd we have

$$
K_{n}(0, b ; p)=\left(\frac{b}{p}\right) \tau_{p}
$$

for example. These formulae yield the remaining parts of the lemma.

We end this section with a result for $q=p^{2}$.

Lemma 27 Suppose that $F(\mathbf{x})=F^{(0)}(\mathbf{x})$ and $p \nmid \Delta$. Then $S_{p^{2}}(\mathbf{c})=0$ unless $p \mid M^{-1}(\mathbf{c})$. Moreover

$$
S_{p^{2}}(\mathbf{c})=p^{n+1}(p-1)
$$

whenever $p^{2} \mid M^{-1}(\mathbf{c})$.

We prove this using Lemma 24. The condition $p \mid d \nabla F(\mathbf{x})+\mathbf{c}$ becomes $p \mid 2 d M \mathbf{x}+\mathbf{c}$, whence $\left.\mathbf{x} \equiv-\overline{2 d} M^{-1} \boldsymbol{\phi m o d} p\right)$. It follows that

$$
F(\mathbf{x})=\mathbf{x}^{T} M \mathbf{x} \equiv \overline{4 d^{2}} M^{-1}(\mathbf{c})(\bmod p),
$$

so that $\Sigma^{(1)}$ is empty if $p / M^{-1}(\mathbf{c})$. If $p^{2} \mid M^{-1}(\mathbf{c})$ we write

$$
\mathbf{x}=-\overline{2 d} M^{-1} \mathbf{c}+p \mathbf{y}
$$

and find that

$$
d \mathbf{x}^{T} M \mathbf{x}+\mathbf{x} \cdot \mathbf{c} \equiv-\overline{4 d} M^{-1}(\mathbf{c})\left(\bmod p^{2}\right) .
$$

Thus $\Sigma^{(1)}$ has a single term, whose value is 1 . The result then follows. 


\section{Averages of $S_{q}(\mathbf{c})$}

In due course we will have to estimate sums of the form $\sum q^{-n} S_{q}(\mathbf{c}) I_{q}(\mathbf{c})$ and we therefore begin by investigating $\sum\left|S_{q}(\mathbf{c})\right|$, so that a trivial bound can be applied. Lemma 25 immediately shows that

$$
\sum_{q \leq X}\left|S_{q}(\mathbf{c})\right| \ll_{\Delta} X^{(4+n) / 2},
$$

but in most cases we can do better. If we write $q=u v$ where $u$ is square-free and $v$ is square-full, then Lemmas 23 and 25 yield

$$
S_{q}(\mathbf{c}) \ll_{\Delta} v^{1+n / 2}\left|S_{u}(\bar{v} \mathbf{c})\right| .
$$

Further applications of Lemma 23 allow us to decompose $u$ into its prime factors, so that Lemma 26 may be used. Since $S_{p}(\mathbf{c}) \ll_{\Delta} 1$ for $p \mid \Delta$ we find that

$$
\left|S_{u}(\bar{v} \mathbf{c})\right| \ll A^{\omega(u)} u^{(n+1) / 2}\left(u, m, M^{-1}(\mathbf{c})\right)^{1 / 2},
$$

for an appropriate constant $A=A(\Delta)$. Moreover the final factor on the right may be omitted if $n$ is odd. Now if $k \neq 0$ we have

$$
\sum_{u \leq U}(u, k) \leq \sum_{d \mid k} d \sum_{u \leq U, d \mid u} 1 \leq \sum_{d \mid k} d \frac{U}{d}=U d(k) .
$$

We shall take $|\mathbf{c}| \leq P$, so that the corresponding value of $k$ is $O_{M}\left(P^{2}\right)$. It follows that, unless $n$ is even and $m=M^{-1}(\mathbf{c})=0$, we will have

$$
\begin{aligned}
\sum_{q \leq X}\left|S_{q}(\mathbf{c})\right| & \ll_{\Delta, \varepsilon} \quad X^{(1+n) / 2} \sum_{v \leq X} v^{1 / 2} \sum_{u \leq X / v} u^{\varepsilon}\left(u, m, M^{-1}(\mathbf{c})\right) \\
& \ll_{M, \varepsilon} \quad X^{(3+n) / 2+\varepsilon} P^{\varepsilon} \sum_{v \leq X} v^{-1 / 2} \\
& \ll_{M, \varepsilon} \quad X^{(3+n) / 2+2 \varepsilon} P^{\varepsilon},
\end{aligned}
$$

on using the fact that there are $O\left(V^{1 / 2}\right)$ square-full numbers $v \leq V$. We therefore conclude as follows.

Lemma 28 Let $|\mathbf{c}| \leq P$. Then for any $\varepsilon>0$ we have

$$
\sum_{q \leq X}\left|S_{q}(\mathbf{c})\right| \ll_{M, \varepsilon} X^{(3+n) / 2+\varepsilon} P^{\varepsilon},
$$

except when $n$ is even and $m=M^{-1}(\mathbf{c})=0$, in which case we have

$$
\sum_{q \leq X}\left|S_{q}(\mathbf{c})\right| \ll_{\Delta} X^{(4+n) / 2} .
$$


This estimate suffices for our purposes, except when $n=4$ and $m=$ $M^{-1}(\mathbf{c})=0$, or when $n=3$ and $m=0$. We proceed to examine these exceptional cases in more detail. We begin by introducing the Dirichlet series

$$
\zeta(s, \mathbf{c})=\sum_{q=1}^{\infty} q^{-s} S_{q}(\mathbf{c})
$$

In view of Lemma 28 this converges absolutely for $\sigma>2+n / 2$. In order to express our function as a product of Euler factors we will need a multiplicativity property of the type given in Lemma 23, but in which $\mathbf{c}$ does not change. Since $m=0$ for the cases under consideration, we have, for any $k$ coprime to $q$,

$$
\begin{aligned}
S_{q}(\mathbf{c}) & =\sum_{a(\bmod q)} * \sum_{\mathbf{b}(\bmod q)} e_{q}(a F(\mathbf{b})+\mathbf{b . c}) \\
& =\sum_{g(\bmod q)} * \sum_{\mathbf{h}(\bmod q)} e_{q}(g F(\mathbf{h})+k \mathbf{h} . \mathbf{c}) \\
& =S_{q}(k \mathbf{c})
\end{aligned}
$$

via the substitutions $\mathbf{b}=k \mathbf{h}$ and $k^{2} a=g$. Here we use the fact that $F(k \mathbf{h})=$ $k^{2} F(\mathbf{h})$ in the homogeneous case. Lemma 23 therefore produces

$$
S_{u v}(\mathbf{c})=S_{u}(\mathbf{c}) S_{v}(\mathbf{c})
$$

whenever $(u, v)=1$, whence

$$
\zeta(s, \mathbf{c})=\prod_{p}\left\{\sum_{t=0}^{\infty} p^{-t s} S_{p^{t}}(\mathbf{c})\right\} .
$$

We proceed to examine the behaviour of the factors above. We begin by observing that the infinite sum will be absolutely convergent for $\sigma>1+n / 2$, by virtue of Lemma 25. This will suffice to handle the prime factors of $\Delta$, since there are only a bounded number of these.

For the remaining factors we handle separately the cases $n=3$ and $n=4$. When $n=3$ and $m=0$ we begin by observing that the terms for $t \geq 3$ contribute $O_{\Delta}\left(p^{-1-3 \delta}\right)$ when

$$
\sigma \geq \frac{17}{6}+\delta
$$

by Lemma 25 . When $p \nmid M^{-1}(\mathbf{c})$ Lemma 27 yields $S_{p^{2}}(\mathbf{c})=0$. If we set

$$
\chi_{\mathbf{c}}(p)=\left(\frac{-\operatorname{det}(M) M^{-1}(\mathbf{c})}{p}\right),
$$

this being a character to modulus $4 \Delta\left|M^{-1}(\mathbf{c})\right|$, it follows from Lemma 26 that

$$
\sum_{t=0}^{\infty} p^{-t s} S_{p^{t}}(\mathbf{c})=1+\chi_{\mathbf{c}}(p) p^{2-s}+O_{\Delta}\left(p^{-1-3 \delta}\right)
$$


for $p \nmid M^{-1}(\mathbf{c})$. For the remaining primes, Lemma 25 yields

$$
\sum_{t=0}^{\infty} p^{-t s} S_{p^{t}}(\mathbf{c})=1+\chi_{\mathbf{c}}(p) p^{2-s}+O_{\Delta}\left(p^{-2 / 3-2 \delta}\right) .
$$

It follows that

$$
\left\{1-\chi_{\mathbf{c}}(p) p^{2-s}\right\}\left\{\sum_{t=0}^{\infty} p^{-t s} S_{p^{t}}(\mathbf{c})\right\}=1+O_{\Delta}\left(p^{-1-2 \delta}\right)
$$

for $p \nmid M^{-1}(\mathbf{c})$, while for $p \mid M^{-1}(\mathbf{c})$ we get $1+0_{\Delta}\left(p^{-2 / 3-2 \delta}\right)$. We therefore deduce that if $M^{-1}(\mathbf{c}) \neq 0$, then

$$
\zeta(s, \mathbf{c})=L\left(s-2, \chi_{\mathbf{c}}\right) \nu(s, \mathbf{c})
$$

where

$$
\nu(s, \mathbf{c}) \ll_{\delta, \varepsilon, M}|\mathbf{c}|^{\varepsilon}, \quad\left(\sigma \geq \frac{17}{6}+\delta\right) .
$$

There remains the possibility that $M^{-1}(\mathbf{c})=0$. In this case Lemmas 25,26 and 27 show that

$$
\sum_{t=0}^{\infty} p^{-t s} S_{p^{t}}(\mathbf{c})=1+p^{4-2 s}(p-1)+O_{\Delta}\left(p^{-1-3 \delta}\right)=1+p^{5-2 s}+O_{\Delta}\left(p^{-1-2 \delta}\right)
$$

for $\sigma$ in the range (11.1). It therefore follows, as in the proof of (11.2), that

$$
\zeta(s, \mathbf{c})=\zeta(2 s-5) \nu(s, \mathbf{c}),
$$

with

$$
\nu(s, \mathbf{c}) \ll_{\delta, M} 1, \quad\left(\sigma \geq \frac{17}{6}+\delta\right) .
$$

We turn now to the case in which $n=4$ and $m=M^{-1}(\mathbf{c})=0$. Here we see that the terms with $t \geq 2$ contribute $O_{\Delta}\left(p^{-1-2 \delta}\right)$ for

$$
\sigma \geq \frac{7}{2}+\delta
$$

by Lemma 25 . In view of Lemma 26 we now see that the Euler factor is

$$
1+\chi(p) p^{2-s}(p-1)+O_{\Delta}\left(p^{-1-2 \delta}\right)
$$

where

$$
\chi(p)=\left(\frac{\operatorname{det}(M)}{p}\right)
$$

is a character modulo $4 \Delta$. Since

$$
\left\{1-\chi(p) p^{3-s}\right\}\left\{1+\chi(p) p^{2-s}(p-1)+O_{\Delta}\left(p^{-1-2 \delta}\right)\right\}=1+O_{\Delta}\left(p^{-1-\delta}\right),
$$


we find that

$$
\zeta(s, \mathbf{c})=L(s-3, \chi) \nu(s, \mathbf{c})
$$

where

$$
\nu(s, \mathbf{c}) \ll_{\delta, M} 1, \quad\left(\sigma \geq \frac{7}{2}+\delta\right) .
$$

We now summarize all our results.

Lemma 29 Write

$$
\zeta(s, \mathbf{c})=\sum_{q=1}^{\infty} q^{-s} S_{q}(\mathbf{c})
$$

and suppose that $n=3$ and $m=0$. Then $\zeta(s, \mathbf{c})$ has an analytic continuation to the region $\sigma>17 / 6$. If $M^{-1}(\mathbf{c}) \neq 0$ then

$$
\zeta(s, \mathbf{c})=L\left(s-2, \chi_{\mathbf{c}}\right) \nu(s, \mathbf{c})
$$

where

$$
\chi_{\mathbf{c}}(p)=\left(\frac{-\operatorname{det}(M) M^{-1}(\mathbf{c})}{p}\right)
$$

for all odd primes $p$, and

$$
\nu(s, \mathbf{c}) \ll_{\delta, \varepsilon}|\mathbf{c}|^{\varepsilon}
$$

uniformly for $\sigma \geq 17 / 6+\delta$. Similarly if $M^{-1}(\mathbf{c})=0$ then

$$
\zeta(s, \mathbf{c})=\zeta(2 s-5) \nu(s, \mathbf{c})
$$

with $\nu(s, \mathbf{c})$ as before.

When $n=4$ and $m=M^{-1}(\mathbf{c})=0$ then $\zeta(s, \mathbf{c})$ has an analytic continuation to the region $\sigma>7 / 2$, and

$$
\zeta(s, \mathbf{c})=L(s-3, \chi) \nu(s, \mathbf{c})
$$

where

$$
\chi(p)=\left(\frac{\operatorname{det}(M)}{p}\right)
$$

for all odd primes $p$, and where now (11.3) holds uniformly for $\sigma \geq 7 / 2+\delta$.

We are now in a position to consider the sum

$$
S(X)=\sum_{q \leq X} S_{q}(\mathbf{c})
$$

As before it is only the cases in which $m=0$ and either $n=3$, or $n=4$ and $M^{-1}(\mathbf{c})=0$ which will be of importance. We shall apply Perron's formula, as given by Titchmarsh [19; Lemma 3.12]. We take $X$ to be half an odd integer, and deduce that

$$
S(X)=\frac{1}{2 \pi i} \int_{2+n-i T}^{2+n+i T} \zeta(s, \mathbf{c}) X^{s} \frac{d s}{s}+O\left(T^{-1} X^{2+n}\right) .
$$


We now set $T=X^{4+2 n}$, and move the line of integration back to $\alpha=\frac{17}{6}+\varepsilon$ or $\alpha=\frac{7}{2}+\varepsilon$ as appropriate. To estimate the error involved in doing this we note that

$$
\zeta(s, \mathbf{c}) \ll_{M, \varepsilon} T^{1 / 2}(1+|\mathbf{c}|)
$$

along the relevent lines, since the characters $\chi$ and $\chi_{\mathbf{c}}$ have moduli $O_{\Delta}(1)$ and $O_{\Delta}\left(\left|M^{-1}(\mathbf{c})\right|\right)=O_{M}\left(|\mathbf{c}|^{2}\right)$ respectively. It follows that

$$
\zeta(s, \mathbf{c}) X^{s} s^{-1} \ll_{M, \varepsilon} 1+|\mathbf{c}| .
$$

We also observe that

$$
\int_{-T}^{T}|\zeta(\alpha+i t, \mathbf{c})| \frac{d t}{1+|t|} \ll_{M, \varepsilon}(1+|\mathbf{c}|) \log T
$$

in view of the mean-value estimate

$$
\int_{0}^{U}|L(\sigma+i t, \chi)|^{2} d t \ll_{\sigma, \varepsilon} U k^{1 / 2+\varepsilon},
$$

valid for any character $\chi(\bmod k)$, and any $\sigma \in\left(\frac{1}{2}, 1\right)$. We therefore find that

$$
S(X)=\operatorname{Res}+O_{M, \varepsilon}\left(X^{\alpha+\varepsilon}(1+|\mathbf{c}|)\right)
$$

where Res denotes the residue from the pole (if any) at $s=3$ (in the case $n=3$ ) or at $s=4$ (in the case $n=4$ ). We conclude as follows.

Lemma 30 Suppose that $m=0$ and either $n=3$, or $n=4$ and $M^{-1}(\mathbf{c})=0$. Let $\alpha=\frac{17}{6}+\varepsilon$ or $\alpha=\frac{7}{2}+\varepsilon$ in the two cases. Set

$$
\sigma(\mathbf{c})=\prod_{p} \sigma_{p}(\mathbf{c})
$$

where

$$
\sigma_{p}(\mathbf{c})=\left(1-p^{-1}\right) \sum_{t=0}^{\infty} p^{-n t} S_{p^{t}}(\mathbf{c})
$$

Then

$$
\sum_{q \leq X} S_{q}(\mathbf{c})=\eta(\mathbf{c}) \sigma(\mathbf{c}) \frac{X^{n}}{n}+O_{M, \varepsilon}\left(X^{\alpha}(1+|\mathbf{c}|)\right),
$$

where $\eta(\mathbf{c})=0$ except in the following cases:-

(i) $n=3$ and $-\operatorname{det}(M) M^{-1}(\mathbf{c})$ is a non-zero square, in which case $\eta(\mathbf{c})=1$;

(ii) $n=3$ and $M^{-1}(\mathbf{c})=0$, in which case $\eta(\mathbf{c})=\frac{1}{2}$;

(iii) $n=4$ and $M^{-1}(\mathbf{c})=0$, and $\operatorname{det}(M)$ is a square, in which case $\eta(\mathbf{c})=1$.

Moreover

$$
\sigma(\mathbf{c}) \ll_{M, \varepsilon} 1+|\mathbf{c}|^{\varepsilon}
$$

whenever $\eta(\mathbf{c}) \neq 0$. 
It is the exceptional value of $\eta(\mathbf{c})$ in case (ii) above which gives rise to the Tamagawa number 2 in Corollary 2.

We conclude this section with some remarks about the sum

$$
\sum_{q \leq X} q^{-n} S_{q}(\mathbf{0})
$$

this being the 'singular series'. When $n \geq 5$, or when $n=4$ and $m \neq 0$, the corresponding infinite sum is absolutely convergent, and Lemma 28 yields

$$
\sum_{q \leq X} q^{-n} S_{q}(\mathbf{0})=\sum_{q=1}^{\infty} q^{-n} S_{q}(\mathbf{0})+O_{M, \varepsilon}\left(X^{(3+\delta-n) / 2+\varepsilon} P^{\varepsilon}\right),
$$

where $\delta=1$ if $n$ is even and $m=0$, and $\delta=0$ otherwise. Moreover $q^{-n} S_{q}(\mathbf{0})$ is a multiplicative function of $q$, by Lemma 23 . The infinite sum is therefore given by

$$
\prod_{p} \sigma_{p}
$$

where

$$
\sigma_{p}=\sum_{t=0}^{\infty} p^{-n t} S_{p^{t}}(\mathbf{0}) .
$$

In the remaining cases, in which $m=0$ and $n=3$ or 4 , we can use Perron's formula, as in the proof of Lemma 30, to show that

$$
\sum_{q \leq X} q^{-n} S_{q}(\mathbf{0})=\operatorname{Res}+O_{M, \varepsilon}\left(X^{\alpha-n+\varepsilon}\right),
$$

where Res is the residue of $\zeta(n+s, \mathbf{0}) X^{s} / s$ at $s=0$. When $n=4, m=0$ and $\operatorname{det}(M)$ is a non-square it follows that

$$
\sum_{q \leq X} q^{-n} S_{q}(\mathbf{0})=\zeta(4, \mathbf{0})+O_{M, \varepsilon}\left(X^{-1 / 2+2 \varepsilon}\right) .
$$

Here $\zeta(4, \mathbf{0})$ can be written as

$$
L(1, \chi) \nu(4, \mathbf{0})=L(1, \chi) \sigma^{*}(F),
$$

where

$$
\chi(p)=\left(\frac{\operatorname{det}(M)}{p}\right)
$$

for all odd primes $p$, and

$$
\sigma^{*}(F)=\prod_{p} \sigma_{p}^{\prime}
$$

with

$$
\sigma_{p}^{\prime}=\left\{1-\chi(p) p^{-1}\right\} \sigma_{p}
$$


In the other cases $\zeta(n+s, \mathbf{0}) X^{s} / s$ has a second order pole at $s=0$, and the residue takes the form

$$
\eta(\mathbf{0}) \sigma(\mathbf{0}) \log X+\sigma^{*} .
$$

Here $\sigma^{*}$ is a constant, depending solely on $M$. Moreover

$$
\sigma(\mathbf{0})=\prod_{p} \sigma_{p}^{\prime}
$$

where

$$
\sigma_{p}^{\prime}=\left\{1-p^{-1}\right\} \sigma_{p}
$$

We summarize these findings as follows.

Lemma 31 When $n \geq 5$, or when $n=4$ and $m \neq 0$, we have

$$
\sum_{q \leq X} q^{-n} S_{q}(\mathbf{0})=\prod_{p} \sigma_{p}+O_{M, \varepsilon}\left(X^{(3+\delta-n) / 2+\varepsilon} P^{\varepsilon}\right)
$$

where $\sigma_{p}$ is given by (11.4) and $\delta=1$ if $n$ is even and $m=0$, and $\delta=0$ otherwise. When $n=4, m=0$ and $\operatorname{det}(M)$ is a non-square, we have

$$
\sum_{q \leq X} q^{-n} S_{q}(\mathbf{0})=L(1, \chi) \sigma^{*}(F)+O_{M, \varepsilon}\left(X^{-1 / 2+\varepsilon}\right)
$$

where $\chi$ is given by (11.5) and $\sigma^{*}(F)$ is given by (11.6) and (11.7). When $n=4, m=0$ and $\operatorname{det}(M)$ is a square, or when $n=3$ and $m=0$, we have

$$
\sum_{q \leq X} q^{-n} S_{q}(\mathbf{0})=\eta(\mathbf{0}) \sigma^{*}(F) \log X+\sigma^{*}+O_{M, \varepsilon}\left(X^{\alpha-n}\right)
$$

with $\sigma^{*}(F)$ given by (11.6) and (11.8).

It follows from what we have said that $\hat{\sigma}$, given by $\prod \sigma_{p}$, (for $n \geq 5$ or $n=$ $4, m \neq 0$ ) or $\prod \sigma_{p}^{\prime}$ (in the remaining cases), is necessarily absolutely convergent. Consequently $\hat{\sigma}$ is real and strictly positive, providing that each of the factors is. We therefore have to consider the sum

$$
\sigma_{p}=\sum_{t=0}^{\infty} p^{-n t} S_{p^{t}}(\mathbf{0})
$$

in more detail. However, as in the usual analysis of the singular series we find that

$$
\begin{aligned}
\sigma_{p} & =\lim _{k \rightarrow \infty} \sum_{t=0}^{k} p^{-n t} S_{p^{t}}(\mathbf{0}) \\
& =\lim _{k \rightarrow \infty} p^{-(n-1) k} \#\left\{\mathbf{x}\left(\bmod p^{k}\right): F(\mathbf{x}) \equiv 0\left(\bmod p^{k}\right)\right\}
\end{aligned}
$$

and the limit is positive providing that there is a non-singular $p$-adic solution. Since the underlying quadratic form in our problem is non-singular we deduce that $\hat{\sigma}>0$ providing that the equation $F=0$ is solvable in every $p$-adic field. 


\section{The Easy Cases-Theorems 4, 5 and 6}

In this section we shall handle those cases for which either Lemma 28 suffices, or the exceptional cases of Lemma 30 do not occur. We begin by observing that, according to Lemmas 19 and 25, we have

$$
\sum_{|\mathbf{c}|>P^{\varepsilon}} \sum_{q=1}^{\infty} q^{-n} S_{q}(\mathbf{c}) I_{q}(\mathbf{c}) \ll_{\varepsilon, S} 1,
$$

since $I_{q}(\mathbf{c})=0$ for $q \gg P$. It therefore suffices to restrict attention to $\mathbf{c} \ll P^{\varepsilon}$. Here we shall use Lemma 22, which yields

$$
I_{q}(\mathbf{c}) \ll_{S, \varepsilon} P^{n / 2+1+\varepsilon} q^{n / 2-1}
$$

for any $\mathbf{c} \neq \mathbf{0}$. In order to combine this with Lemma 28 it will be convenient to write, as before, $\delta=1$ if $n$ is even and $m=0$, and $\delta=0$ otherwise. With this notation it follows that

$$
\sum_{R<q \leq 2 R} q^{-n} S_{q}(\mathbf{c}) I_{q}(\mathbf{c}) \ll_{S, \varepsilon} P^{n / 2+1+\varepsilon} R^{(1+\delta) / 2+\varepsilon}
$$

for any $R$, whence

$$
\sum_{q=1}^{\infty} q^{-n} S_{q}(\mathbf{c}) I_{q}(\mathbf{c}) \ll_{S, \varepsilon} P^{(n+3+\delta) / 2+2 \varepsilon} .
$$

We may now sum for non-zero values of $\mathbf{c}$ in the range $\mathbf{c} \ll P^{\varepsilon}$ and deduce that

$$
\sum_{\mathbf{c} \neq \mathbf{0}} \sum_{q=1}^{\infty} q^{-n} S_{q}(\mathbf{c}) I_{q}(\mathbf{c}) \ll_{S, \varepsilon} P^{(n+3+\delta) / 2+(n+2) \varepsilon} .
$$

It remains to consider $\mathbf{c}=\mathbf{0}$. For $q>Q P^{-\varepsilon}$ we use Lemma 16 in conjunction with Lemma 28 to show that

$$
\sum_{R<q \leq 2 R} q^{-n} S_{q}(\mathbf{0}) I_{q}(\mathbf{0}) \ll_{S, \varepsilon} P^{n+\varepsilon} R^{(3+\delta-n) / 2+\varepsilon}
$$

for any $R$, whence

$$
\sum_{q>Q P^{-\varepsilon}} q^{-n} S_{q}(\mathbf{0}) I_{q}(\mathbf{0}) \ll_{S, \varepsilon} P^{(n+3+\delta) / 2+2 \varepsilon} .
$$

When $q \leq Q P^{-\varepsilon}$ we can call on Lemmas 13 and 31, which show that

$$
\begin{aligned}
\sum_{q \leq Q P^{-\varepsilon}} q^{-n} S_{q}(\mathbf{0}) I_{q}(\mathbf{0}) & =P^{n} \sigma_{\infty}(w, G) \sum_{q \leq Q P^{-\varepsilon}} q^{-n} S_{q}(\mathbf{0})+O_{S, \varepsilon}(1) \\
& =P^{n} \sigma_{\infty}(w, G) \prod_{p} \sigma_{p}+O_{S, \varepsilon}\left(P^{(n+3+\delta) / 2+n \varepsilon}\right) .
\end{aligned}
$$


These estimates suffice for the proof of Theorems 4 and 5 .

We turn now to Theorem 6 . Here we shall save the factor $X^{1 / 2}$ which is lost in Lemma 28 when $M^{-1}(\mathbf{c})=0$, by using Lemma 30 instead. This requires us to remove the factor $I_{q}(\mathbf{c})$ by partial summation. In view of Lemmas 19, 22 and 28 the terms with $|\mathbf{c}|>P^{\varepsilon}$ or with $M^{-1}(\mathbf{c}) \neq 0$ may be handled as before, producing a contribution $O_{S, \varepsilon}\left(P^{7 / 2+6 \varepsilon}\right)$ to

$$
\sum_{\mathbf{c}} \sum_{q} q^{-4} S_{q}(\mathbf{c}) I_{q}(\mathbf{c})
$$

When $0<|\mathbf{c}| \leq P^{\varepsilon}$ and $M^{-1}(\mathbf{c})=0$ we may use partial summation, based on Lemmas 30 and 22, to show that

$$
\sum_{R<q \leq 2 R} q^{-4} S_{q}(\mathbf{c}) I_{q}(\mathbf{c}) \ll_{S, \varepsilon} P^{3+\varepsilon} R^{1 / 2+\varepsilon} .
$$

These values of $\mathbf{c}$ may therefore be handled as before too. For $\mathbf{c}=\mathbf{0}$ we again use partial summation, based on Lemmas 30 and 16 this time, to obtain

$$
\sum_{R<q \leq 2 R} q^{-4} S_{q}(\mathbf{0}) I_{q}(\mathbf{0}) \ll_{S, \varepsilon} P^{4+\varepsilon} R^{-1 / 2+\varepsilon}
$$

for any $R$. This allows values $q>Q P^{-\varepsilon}$ to be handled as before. For $q \leq Q P^{-\varepsilon}$ we can apply Lemmas 13 and 31, which yield

$$
\begin{aligned}
\sum_{q \leq Q P^{-\varepsilon}} q^{-4} S_{q}(\mathbf{c}) I_{q}(\mathbf{c}) & =P^{4} \sigma_{\infty}(w, G) \sum_{q \leq Q P^{-\varepsilon}} q^{-4} S_{q}(\mathbf{0})+O_{S, \varepsilon}(1) \\
& =P^{4} \sigma_{\infty}(w, G) L(1, \chi) \sigma^{*}(F)+O_{S, \varepsilon}\left(P^{7 / 2+2 \varepsilon}\right) .
\end{aligned}
$$

This suffices for Theorem 6 .

\section{Harder Cases-Theorems 7 and 8}

Our analysis of Theorems 7 and 8 begins as before with the observation that

$$
\sum_{|\mathbf{c}|>P^{\varepsilon}} \sum_{q=1}^{\infty} q^{-n} S_{q}(\mathbf{c}) I_{q}(\mathbf{c}) \ll_{\varepsilon} 1 .
$$

We next treat those cases $|\mathbf{c}| \leq P^{\varepsilon}$ for which $\mathbf{c} \neq \mathbf{0}$. Here Lemma 22 yields

$$
I_{q}(\mathbf{c}) \ll_{S, \varepsilon} P^{n / 2+1+\varepsilon} q^{n / 2-1}
$$

and

$$
\frac{\partial I_{q}(\mathbf{c})}{\partial q} \ll_{S, \varepsilon} P^{n / 2+1+\varepsilon} q^{n / 2-2} .
$$


Now partial summation, based on Lemma 30 yields

$$
\begin{aligned}
\sum_{R<q \leq 2 R} q^{-n} S_{q}(\mathbf{c}) I_{q}(\mathbf{c})=\eta(\mathbf{c}) \sigma(\mathbf{c}) \int_{R}^{2 R} & q^{-1} I_{q}(\mathbf{c}) d q \\
& +O_{S, \varepsilon}\left(P^{n / 2+1+2 \varepsilon} R^{\alpha-n / 2-1+\varepsilon}\right)
\end{aligned}
$$

and hence

$$
\begin{aligned}
\sum_{q=1}^{\infty} q^{-n} S_{q}(\mathbf{c}) I_{q}(\mathbf{c})= & \eta(\mathbf{c}) \sigma(\mathbf{c}) \int_{0}^{\infty} q^{-1} I_{q}(\mathbf{c}) d q \\
& +O_{S, \varepsilon}\left(P^{n / 2+1+2 \varepsilon} P^{\alpha-n / 2-1+\varepsilon}\right) .
\end{aligned}
$$

The integral here is

$$
P^{n} \int_{0}^{\infty} r^{-1} I_{r}^{*}(\mathbf{c}) d r=P^{n} \sigma_{\infty}(F, w, \mathbf{c})
$$

say, and we observe that $\sigma_{\infty}(F, w, \mathbf{c})$ depends only on $F, w$ and $\mathbf{c}$. We proceed to verify that the integral is indeed convergent, as has been tacitly assumed above. Lemmas 14, 18 and 22 yield

$$
I_{r}^{*}(\mathbf{c}) \ll_{S} r^{-1}\left|I\left(r ; r^{-1} \mathbf{c}\right)\right| \ll_{S, N} r^{-1-N}\left(r^{-1}|\mathbf{c}|\right)^{-N}=r^{-1}|\mathbf{c}|^{-N}
$$

and

$$
I_{r}^{*}(\mathbf{c}) \ll_{S} r^{-1}\left|I\left(r ; r^{-1} \mathbf{c}\right)\right| \ll_{S, \varepsilon}\left(r^{-2}|\mathbf{c}|\right)^{\varepsilon}\left(r^{-1}|\mathbf{c}|\right)^{1-n / 2} .
$$

We use the first bound for $r \geq|\mathbf{c}|^{-N / 2}$, and the second for the remaining range. This produces a bound

$$
\sigma_{\infty}(F, w, \mathbf{c}) \ll_{S, \varepsilon, N}|\mathbf{c}|^{-M}
$$

with

$$
M=\min \left\{\frac{N}{2}\left(\frac{n}{2}-2 \varepsilon-1\right)-1+\frac{n}{2}-\varepsilon, \frac{N}{2}\right\} .
$$

This can be made as large as desired, by appropriate choice of $N$. It now follows from (13.1) and (13.2) that

$$
\begin{aligned}
\sum_{\mathbf{c} \neq \mathbf{0}} \sum_{q=1}^{\infty} q^{-n} S_{q}(\mathbf{c}) I_{q}(\mathbf{c}) & =P^{n} \sum_{0<|\mathbf{c}| \leq P^{\varepsilon}} \sigma_{\infty}(F, w, \mathbf{c}) \eta(\mathbf{c}) \sigma(\mathbf{c}) \\
& +O_{S, \varepsilon}\left(P^{\alpha+(n+3) \varepsilon}\right) \\
& =P^{n} \sum_{\mathbf{c} \neq \mathbf{0}} \sigma_{\infty}(F, w, \mathbf{c}) \eta(\mathbf{c}) \sigma(\mathbf{c})+O_{S, \varepsilon}\left(P^{\alpha+(n+3) \varepsilon}\right),
\end{aligned}
$$

in view of (13.3). 
There remains the case $\mathbf{c}=\mathbf{0}$. Here we shall choose a parameter $\rho \leq 1$ and use Lemmas 13, 25 and 31 for $q \leq \rho P$ to give

$$
\begin{aligned}
\sum_{q \leq \rho P} q^{-n} S_{q}(\mathbf{0}) I_{q}(\mathbf{0})=P^{n} \sigma_{\infty}( & F, w)\left\{\eta(\mathbf{0}) \sigma^{*}(F) \log \rho P+\sigma^{*}\right\} \\
& +O_{S, \varepsilon}\left(P^{\alpha} \rho^{\alpha-n}\right)+O_{S, N}\left(P^{n+1 / 2} \rho^{N}\right) .
\end{aligned}
$$

For the remaining range we use partial summation, based on Lemmas 16 and 30 , to show that

$$
\sum_{q>\rho P} q^{-n} S_{q}(\mathbf{0}) I_{q}(\mathbf{0})=\eta(\mathbf{0}) \sigma^{*}(F) \int_{\rho P}^{\infty} q^{-1} I_{q}(\mathbf{0}) d q+O_{S, \varepsilon}\left(P^{\alpha} \rho^{\alpha-n}\right) .
$$

Here we have used the observation that $\sigma(\mathbf{0})=\sigma^{*}(F)$. On combining these estimates, and substituting $I_{q}(\mathbf{0})=P^{n} I_{r}^{*}(\mathbf{0})$, where $r=q / Q$, we obtain

$$
\begin{array}{r}
\sum_{q=1}^{\infty} q^{-n} S_{q}(\mathbf{0}) I_{q}(\mathbf{0})=\eta(\mathbf{0}) \sigma_{\infty}(F, w) \sigma^{*}(F) P^{n} \log P+K(\rho) P^{n} \\
+O_{S, \varepsilon}\left(P^{\alpha} \rho^{\alpha-n}\right)+O_{S, N}\left(P^{n+1 / 2} \rho^{N}\right),
\end{array}
$$

where

$$
K(\rho)=\eta(\mathbf{0}) \sigma^{*}(F)\left\{\sigma_{\infty}(F, w) \log \rho+\int_{\rho}^{\infty} r^{-1} I_{r}^{*}(\mathbf{0}) d r\right\}+\sigma_{\infty}(F, w) \sigma^{*} .
$$

We shall show that $K(\rho)$ tends to a limit as $\rho$ tends to 0 . The limit, which we shall write as $K(0)$, by abuse of notation, is clearly independent of $P$. Moreover we will have $K(\rho)=K(0)+O_{S, N}\left(\rho^{N}\right)$ for any $N>0$. To prove these assertions it suffices to observe that if $0<\rho_{1}<\rho_{2}<1$ then

$$
\begin{aligned}
K\left(\rho_{2}\right)-K\left(\rho_{1}\right) & =\eta(\mathbf{0}) \sigma^{*}(F)\left\{\sigma_{\infty}(F, w) \log \rho_{2} / \rho_{1}-\int_{\rho_{1}}^{\rho_{2}} r^{-1} I_{r}^{*}(\mathbf{0}) d r\right\} \\
& \ll_{S, N} \rho_{2}^{N},
\end{aligned}
$$

by Lemma 13. It now follows that

$$
\begin{array}{r}
\sum_{q=1}^{\infty} q^{-n} S_{q}(\mathbf{0}) I_{q}(\mathbf{0})=\quad \eta(\mathbf{0}) \sigma_{\infty}(F, w) \sigma^{*}(F) P^{n} \log P+K(0) P^{n} \\
+O_{S, \varepsilon}\left(P^{\alpha} \rho^{\alpha-n}\right)+O_{S, N}\left(P^{n+1 / 2} \rho^{N}\right) .
\end{array}
$$

We therefore take $\rho=P^{-\varepsilon}$, and choose $N$ sufficiently large to make the error terms $O_{S, \varepsilon}\left(P^{\alpha+\varepsilon}\right)$.

If we combine this with our results for the case $\mathbf{c} \neq \mathbf{0}$ we see that Theorems 7 and 8 now follow from Theorem 2, on setting

$$
\sigma_{1}(F, w)=K(0)+\sum_{\mathbf{c} \neq \mathbf{0}} \sigma_{\infty}(F, w, \mathbf{c}) \eta(\mathbf{c}) \sigma(\mathbf{c})
$$

and redefining $\varepsilon$. 


\section{Proof of Corollaries 1 and 2}

Corollary 1 requires little comment. We choose

$$
w(\mathbf{x})=e w_{0}(2 G(\mathbf{x})),
$$

where $w_{0}$ is given by $(2.1)$. Thus, since $F^{(0)}$ is positive definite, $w$ is supported in a hypercube of side depending only on $F^{(0)}$. It follows that $w \in \mathcal{C}\left(F^{(0)}\right)$. Moreover $w(\mathbf{x})=1$ whenever $G(\mathbf{x})=0$, and $|G| \leq 1 / 2$ on $\operatorname{supp}(w)$, so that, as noted in the introduction, the non-singularity condition holds automatically. Our choice of $w$ yields

$$
N(F, w)=\#\left\{\mathbf{x} \in \mathbb{Z}^{n}: F^{(0)}(\mathbf{x})=m\right\},
$$

and $\sigma_{\infty}(G, w)=\sigma_{\infty}(G)$, since $w(\mathbf{x})=1+O(\varepsilon)$ for $|G(\mathbf{x})| \leq \varepsilon$. The corollary then follows.

To prove Corollary 2 we observe firstly that

$$
\sum_{k \leq x} \mu(k) \ll x \exp \{-c \sqrt{\log x}\},
$$

for an appropriate absolute constant $c>0$. This is a well-known consequence of the Prime Number Theorem. Since

$$
\sum_{k=1}^{\infty} \frac{\mu(k)}{k}=0
$$

and

it follows via partial summation that

$$
\sum_{k=1}^{\infty} \frac{\mu(k) \log k}{k}=-1
$$

$$
\sum_{k \leq K} \frac{\mu(k)}{k} \ll \exp \{-c \sqrt{\log K}\}
$$

and

$$
\sum_{k \leq K} \frac{\mu(k) \log k}{k}=-1+O(\exp \{-c \sqrt{\log K}\}),
$$

with a new value of $c$. If we insert these estimates into Theorem 8 we find that

$$
\begin{aligned}
\sum_{k \leq K} \mu(k) N(F, w, P / k) & \\
= & \frac{1}{2} \sigma_{\infty}(F, w) \sigma^{*}(F) \sum_{k \leq K} \mu(k) \frac{P}{k}\left\{\log \frac{P}{k}+\sigma_{1}(F, w)\right\} \\
& +O_{S, \varepsilon}\left(P^{5 / 6+\varepsilon} K^{1 / 6}\right) \\
= & \frac{1}{2} \sigma_{\infty}(F, w) \sigma^{*}(F) P+O_{S}(P(\log P) \exp \{-c \sqrt{\log K}\}) \\
& +O_{S, \varepsilon}\left(P^{5 / 6+\varepsilon} K^{1 / 6}\right) .
\end{aligned}
$$


We shall choose $K=\sqrt{P}$, making the above error terms $O_{S}\left(P \exp \left\{-\frac{c}{2} \sqrt{\log P}\right\}\right)$.

To handle values of $k$ bigger than $K$, we first note that $w(\mathbf{x})=0$ if $\mathbf{x}$ is small enough in terms of $S$. This follows from our assumption that $|\nabla F(\mathbf{x})| \gg_{S} 1$ on $\operatorname{supp}(w)$. We therefore see that $N(F, w, P / k)=0$ if $k \gg_{S} P$, so that it remains to investigate the range $K<k \ll_{S} P$. With this in mind we take $K \leq L \ll_{S} P$ and consider

$$
\sum_{L<k \leq 2 L} \mu(k) N(F, w, P / k)=\sum_{F(\mathbf{x})=0} \sum_{L<k \leq 2 L} \mu(k) w\left(\frac{k}{P} \mathbf{x}\right) .
$$

The innermost sum vanishes unless $P L^{-1} \ll_{S}|\mathbf{x}| \ll_{S} P L^{-1}$, in which case partial summation, based on (14.1), produces an estimate

$$
\sum_{L<k \leq 2 L} \mu(k) w\left(\frac{k}{P} \mathbf{x}\right) \ll_{S} L \exp \{-c \sqrt{\log L}\} \ll_{S} L \exp \{-c \sqrt{\log K}\} .
$$

A further application of Theorem 8, with a weight whose support includes the region $P L^{-1} \ll_{S}|\mathbf{x}| \ll_{S} P L^{-1}$, shows that there are $O_{S}\left(P L^{-1} \log P\right)$ possible values of $\mathbf{x}$, whence

$$
\sum_{L<k \leq 2 L} \mu(k) N(F, w, P / k) \ll_{S} P(\log P) \exp \{-c \sqrt{\log K}\} .
$$

We therefore conclude that

$\sum_{k>K} \mu(k) N(F, w, P / k) \ll_{S} P(\log P)^{2} \exp \{-c \sqrt{\log K}\} \ll_{S} P \exp \left\{-\frac{c}{2} \sqrt{\log P}\right\}$,

and Corollary 2 follows.

\section{Acknowledgements}

It is a pleasure to record the support of the Isaac Newton Institute, Cambridge, where much of this work was carried out. In addition thanks are due to Dr B.Z. Moroz for a number of helpful comments on an earlier draft of this paper.

\section{References}

[1] P. Deligne, La conjecture de Weil. I, Publ. Math. IHES, 43 (1973), 273-307.

[2] W. Duke, Hyperbolic distribution problems and half-integral weight Maass forms, Invent. Math., 92 (1988), 73-90.

[3] W. Duke, Lattice points on ellipsoids, Séminaire de théorie des nombres, (Talence, 1987-88) Exp. no. 37, 7pp.

[4] W. Duke, J. Friedlander and H. Iwaniec, Bounds for automorphic Lfunction, Invent. Math., 112 (1993), 1-8. 
[5] D.R. Heath-Brown, Cubic forms in ten variables, Proc. London Math Soc. (3), 47 (1983), 225-257.

[6] C. Hooley, On a new approach to various problems of Waring's type, Recent progress in analytic number theory, Vol I, 127-191 (Academic Press, London, 1981).

[7] C. Hooley, Some recent advances in analytical number theory, Proc. Internat. Congress Math. 1983, 85-97 (North-Holland, Amsterdam, 1984).

[8] C. Hooley, On Waring's problem, Acta Math., 157 (1986), 49-97.

[9] C. Hooley, On nonary cubic forms, J. Reine Angew. Math., 386 (1988), 32-98.

[10] H. Iwaniec, Fourier coefficients of modular forms of half-integral weight, Invent. Math., 87 (1987), 385-401.

[11] H. Iwaniec, The circle method and the Fourier coefficients of modular forms, Number theory and related topics, 47-55 (Tata Institute of Fundamental Research, Bombay, 1989).

[12] H.D. Kloosterman, On the representation of numbers in the form $a x^{2}+$ $b y^{2}+c z^{2}+d t^{2}$, Acta Math., 49 (1926), 407-464.

[13] A.V. Malyshev, On the weighted number of integer points on a quadric, Seminars in Mathematics, I, (Consultants Bureau, New York, 1968), 1-30.

[14] L.J. Mordell, Diophantine equations, (Academic Press, London, 1969).

[15] B.Z. Moroz, On the representation of large integers by integral ternary positive definite quadratic forms, Astérisque, 209 (1992), 275-278.

[16] O.T. O'Meara, Introduction to quadratic forms, (Springer, Berlin, 1963).

[17] C.L. Siegel, Equivalence of quadratic forms, Amer. J. Math., 63 (1941), 658-680.

[18] B.R. Srinivasan, The lattice point problem of many dimensional hyperboloids. III, Math. Ann., 160 (1965), 280-311.

[19] E.C. Titchmarsh, The theory of the Riemann Zeta-function, 2nd edition, (Oxford University Press, Oxford, 1986).

[20] R.C. Vaughan, The Hardy-Littlewood method, Cambridge Tracts in Mathematics, 80 (Cambridge University Press, 1981).

[21] G.L. Watson, Integral Quadratic Forms, Cambridge Tracts in Mathematics, 51 (Cambridge University Press, 1960). 